\title{
LIVER PROTEIN EXPRESSION IN NASH MICE ON A HIGH-FAT DIET
}

By

James Varani, Shannon D McClintock, Randall N Knibbs, Isabelle Harber, Dania Zeidan, Mohamed Ali $\mathrm{H}$ Jawad-Makki and Muhammad N Aslam

\section{From}

The Department of Pathology

University of Michigan Medical School

Ann Arbor, Ml 48109

${ }^{*}$ Corresponding author:

Muhammad N. Aslam

Department of Pathology

The University of Michigan

1150 W Medical Center Dr

Ann Arbor, MI 48109, USA

Tel: (734) 936-1897

Email: mnaslam@med.umich.edu

Short title: Mitigation of high-fat induced liver injury

Keywords: Aquamin, fibrosis, liver injury, liver cancer, mouse model, NAFLD, NASH, proteomics 


\section{ABSTRACT}

Male MS-NASH mice were maintained on a high-fat diet for 16 weeks with and without red algaederived minerals. Obeticholic acid (OCA) was used as a comparator in the same strain and diet. C57BL/6 mice maintained on a standard (low-fat) rodent chow diet were used as a control. At the end of the in-life portion of the study, body weight, liver weight, liver enzyme levels and liver histology were assessed. Samples obtained from individual livers were subjected to Tandem Mass Tag labeling / mass spectroscopy for protein profile determination. As compared to mice maintained on the low-fat diet, all high-fat-fed mice had increased whole body and liver weight, increased liver enzyme (aminotransferases) levels and widespread steatosis / ballooning hepatocyte degeneration.

Histological evidence for liver inflammation and collagen deposition was also present, but changes were to a lesser extent. A moderate reduction in ballooning degeneration and collagen deposition was observed with mineral supplementation. Control mice on the high-fat diet alone demonstrated multiple protein changes associated with dysregulated fat and carbohydrate metabolism, lipotoxicity and oxidative stress. Cholesterol metabolism and bile acid formation were especially sensitive to diet. In mice receiving multi-mineral supplementation along with the high-fat diet, there was reduced liver toxicity as evidenced by a decrease in levels of several cytochrome P450 enzymes and other oxidantgenerating moieties. Additionally, elevated expression of several keratins was also detected in mineralsupplemented mice. The protein changes observed with mineral supplementation were not seen with OCA. Our previous studies have shown that mice maintained on a high-fat diet for up to 18 months develop end-stage liver injury including hepatocellular carcinoma. Mineral-supplemented mice were substantially protected against tumor formation and other end-state consequences of high-fat feeding. The present study identifies early (16-week) protein changes occurring in the livers of the high-fat dietfed mice, and how the expression of these proteins is influenced by mineral supplementation. These findings help elucidate early protein changes that contribute to end-stage liver injury and potential mechanisms by which dietary minerals may mitigate such damage. 


\section{INTRODUCTION}

Non-alcoholic fatty liver disease (NAFLD) is rapidly becoming the most common cause of liver injury in Western society and throughout the world (1-3). It is estimated that up to $25 \%$ of adults worldwide have some degree of hepatic fat accumulation (4). When fat accumulation is the extent of damage, it is well-tolerated by most people. However, in a subset of individuals, disease progression leads to liver inflammation and hepatocyte injury. Cycles of injury and repair result in measurable collagen deposition in the liver parenchyma, leading eventually to detectable fibrotic changes and, in some cases, to cirrhosis. When steatosis is followed by the tissue damage, this state is referred to as non-alcoholic steatohepatitis (NASH) (5). Formation of liver tumors, including hepatocellular carcinoma, is another common and the most devastating late-stage consequence. Why some individuals with extensive steatosis progress to more serious disease including liver cancer and others do not, remains a critical unanswered question.

The high content of saturated fat and sugar in the typical Western diet is thought to underlie fat accumulation (6-8). Experimental studies in rodents have shown that providing a high-fat or high-fat and -sugar diet leads rapidly to fatty liver infiltration - observable as early as after 8 weeks of feeding (9-11). With animals maintained for longer periods (20-25 weeks), evidence of inflammatory changes, ballooning hepatocyte degeneration and collagen deposition may also be seen. Even after 25 weeks, however, these changes are generally mild. Some studies have reported widespread liver damage (beyond steatosis) at earlier time-points but, typically, liver toxins such as carbon tetrachloride, thioacetamide or alcohol are administered along with the high-fat diet $(12,13)$. Animals with severe genetic anomalies (14) or animals maintained on a toxic, nutrient-restricted diet such as the methionine- and choline-deficient diet (15) may develop severe liver anomalies more rapidly. When a high-fat or high-fat and -sugar diet alone is utilized in "genetically normal" strains, overt liver damage (beyond steatosis) is not routinely observed before 36 weeks of age (16) and a year or more is required for terminal damage - including hepatocellular carcinoma - to be widely manifested $(17,18)$. 
In our own previous studies $(19,20)$, C57BL/6 mice were maintained on a high-fat Western-style diet (HFWD) for up to 18 months. The long feeding period allowed for the development of extensive liver damage in a majority of animals, especially males. In addition to wide-spread steatosis, HFWDfed mice demonstrated extensive perivascular and parenchymal liver inflammation, ballooning hepatocyte injury and collagen deposition. Large fibrotic nodules and areas of infarct were observed in many of the animals. Liver tumors including hepatic adenomas and hepatocellular carcinomas were identified grossly and histologically in many of the animals. Of most interest, our studies $(19,20)$ demonstrated that providing an adequate level of calcium (estimated to be $20-25 \mathrm{mg} /$ day consumed) along with multiple other trace elements in a mineral supplement (Aquamin ${ }^{\circledR}$ ) dramatically reduced tumor formation (20). Inflammation, hepatocyte injury and collagen deposition were also reduced while steatosis, itself, was largely unaffected.

While an extended feeding period is essential to see the full spectrum of liver disease develop in rodents, long-term studies are not optimal for identifying the early events that lead to steatosis and disease progression in high-fat-fed mice. Likewise, long-term studies are not ideal for understanding how dietary minerals (or other interventions) might mitigate disease progression. As a way to help understand the early events that drive liver disease progression and its prevention, cohorts of mice (MS-NASH strain) were placed on a high-fat diet with fructose added to the drinking water (high-fat diet) with and without mineral supplementation $\left(\right.$ Aquamin $\left.^{\circledR}\right)$ for a period of 16 weeks. An additional cohort of high-fat diet mice was treated with obeticholic acid (OCA), a drug that acts as a Farnesoid X Receptor (FXR) agonist, that has been shown to reduce liver damage in both humans (21-24) and mice (25). C57BL/6 mice fed a low-fat rodent chow diet served as an additional control. At the end of the in-life portion of the study, whole body weight and live weight were obtained from each animal along with liver enzyme levels and metabolic parameters. Livers were assessed histologically for steatosis and for other changes (hepatocyte degeneration, inflammation, and collagen deposition) indicative of more serious damage. Finally, liver tissue samples were evaluated using a tandem mass tag (TMT) mass 
spectrometry-based proteomic approach for protein expression levels in individual animals. Findings from these relatively short-term studies (especially the proteomic analyses) allow us to identify early liver changes that reflect diet differences (i.e., that distinguish low-fat and high-fat feeding) and how the effects of high-fat feeding may be modified in response to intervention. Findings from this study are described here. 


\section{MATERIALS AND METHODS}

Experimental diets and interventions. Diet D12079B (Research Diets Inc., New Brunswick, NJ) with 5\% fructose added to the drinking water was used as the high-fat Western diet for this study. This diet was designed to provide $500 \mathrm{gm} / \mathrm{kg}$ of carbohydrates, $198 \mathrm{gm} / \mathrm{kg}$ of proteins and $210 \mathrm{gm} / \mathrm{kg}$ of fat. The percentage of calories from carbohydrates, proteins and fat in this diet was 43,17 , and $40 \%$ respectively. This diet also contained $39 \mathrm{gm} / \mathrm{kg}$ of a standard mineral mix (S10001A) containing calcium carbonate and calcium phosphate and other essential trace elements. Diet 5008 (Purina) was used as the low-fat control diet. The caloric distribution from this low-fat diet was approximately 57,27 , and $16 \%$ from carbohydrates, proteins and fat, respectively. Diet 5008 was supplemented with standard minerals $(2.5 \%)$ as part of the diet-formula. High-fat diet alone was considered as the control for comparison with the low-fat diet or with Aquamin ${ }^{\circledR}$ and OCA as interventions in the high-fat diet.

Aquamin ${ }^{\circledR}$ is a product rich in calcium, magnesium, and trace elements, obtained from the

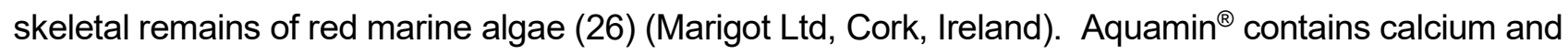
magnesium in an approximately 14:1 ratio, along with measurable levels of 72 additional trace minerals including minerals with calcimimetic activity (for example, magnesium, strontium, and trace elements from the lanthanide family [27-29]), and essentially all of the minerals concentrated from ocean water in the algal fronds. Mineral composition was established by an independent laboratory (Advanced Laboratories; Salt Lake City, Utah) using Inductively Coupled Plasma Optical Emission Spectrometry (ICP-OES). Supplement Table 1 provides a complete list of elements detected in Aquamin ${ }^{\circledR}$ and their relative amounts. Aquamin ${ }^{\circledR}$ is sold as a dietary supplement (GRAS 000028) and is used in various products for human consumption in Europe, Asia, Australia, and North America. A single batch of Aquamin ${ }^{\circledR}$-Soluble (citratemalate salt) was used for this study. Aquamin ${ }^{\circledR}$-Soluble was added to the drinking water $(18 \mathrm{mg} / \mathrm{mL})$ throughout the entire in-life portion of the study. We have used Aquamin ${ }^{\circledR}$ in previously conducted long term mouse studies $(20,30,31)$ 
Obeticholic acid (OCA) was prepared as a $3 \mathrm{mg} / \mathrm{mL}$ suspension in $0.5 \%$ methylcellulose. Animals received $0.25 \mathrm{~mL}$ OCA (approximately $30 \mathrm{mg} / \mathrm{kg}$ ) by oral gavage five times per week beginning on week-8 of high-fat feeding.

Mouse model and in-life study protocol. The study was conducted in a mouse model (MS-NASH mouse, formally known as FATZO $[32,33])$ by Crown Bioscience, Inc. Three cohorts of male MS-NASH mice ( 9 animals per group) were maintained on the high-fat diet, beginning at 6-7 weeks of age (at weaning) and after one week of acclimation. One group was kept as control (high-fat diet alone) while the other groups received Aquamin ${ }^{\circledR}$ or OCA as interventions along with the high-fat Western diet. Since mineral supplementation $\left(\right.$ Aquamin $^{\circledR}$ ) is proposed as a preventive agent, it was included along with high-fat feeding. OCA is envisioned as a therapeutic agent for existing disease, and animals were started on OCA at week-8 (16 weeks of age). A low-fat diet control group consisted of male C57BL/6 (Envigo) mice (9 animals per group) maintained on Diet 5008. Food and water were provided ad libitum. The animal environment was maintained at $70-74^{\circ} \mathrm{F}$ with a $12-$ hour light-dark cycle. During the in-life portion of the study, health-checks were done daily. At termination (after 16 weeks on diet), animals were euthanized by carbon dioxide asphyxiation. The entire in-life portion of the study and termination (euthanasia and tissue harvesting) was carried out by Crown Bio, Inc., at their Lafayette, LA facility under the approved Standard Operating Procedures at the testing site. All procedures involving live animals were approved by the Institutional Animal Care and Use Committee (IACUC). Crown Bio, Inc., is an AAALAC-accredited institution.

At euthanasia, blood was obtained by cardiac puncture and used to assess liver enzyme (alanine aminotransferase [ALT] and aspartate aminotransferase [AST]) levels as a measure of injury and triglyceride (TG) levels as an indicator of metabolic function. Livers were removed and weighed. After gross examination, a piece of tissue from the left lobe was immediately frozen in liquid nitrogen and used for proteomic analysis. Another tissue piece from the left lobe was fixed in $10 \%$ buffered formalin and used for histology. 
AST, ALT and triglyceride assessment. Serum levels of AST, ALT and triglycerides were assessed using a Beckman chemical analyzer. All blood chemistry measurements were made following a standard operating procedure at Crown Bioscience.

Histology and assessment scoring. Formalin-fixed liver tissue samples were processed for histology and 3-4 $\mu$ m-thick sections were stained with hematoxylin and eosin (H\&E) and picrosirius red (PSR), independently. H\&E-stained tissue sections were evaluated under light microscopy for evidence of steatosis, ballooning hepatocyte degeneration and inflammation using the Kleiner scoring system (34). Kleiner et al has described the standardized histological scoring assessments of these NASH-related parameters in liver sections (34). These scoring methods have previously been applied to the histological scoring of mouse models of steatohepatitis by others $(35,36)$ and by us $(19)$. Under this scoring system, a score of (0-3) was given for steatosis and inflammation separately, and a score of (0-2) for ballooning degeneration of hepatocytes. From these evaluations, a NAFLD activity score (NAS) was calculated by adding these individual scores. Finally, PSR-stained liver sections were used to assess collagen deposition and scored using a scale from 0-4 as defined by Kleiner et al (34).

After microscopic evaluation, slides were digitized using the Aperio AT2 whole slide scanner (Leica Biosystems) at $40 x$ with a resolution of $0.5 \mu \mathrm{m}$ per pixel with $20 x$ objective. The scanned images were housed on a server and accessed using Leica Aperio eSlide Manager (Version 12.3.2.5030), a digital pathology management software. These digitized histological sections were viewed and analyzed using Aperio ImageScope (Version 12.3.3.5048), a slide viewing software by Leica.

Proteomic assessment: Proteomic experiments were carried out in the Proteomics Resource Facility (PRF), a core laboratory in the Department of Pathology at the University of Michigan. For protein mass spectrometry analysis, we employed liver tissue from five mice from each group and assessed each separately. A cryopreserved tissue piece from the left lobe (of each mouse liver) was weighed and homogenized in Radioimmuno-Precipitation Assay (RIPA) - lysis and extraction buffer (Pierce, \# 89901; 
ThermoFisher Scientific) for protein isolation, as described in our previous publications (37-39). Fifty micrograms of sample protein from each liver were digested separately with trypsin and individual samples labeled with one of 11 isobaric mass tags following the manufacturer's protocol. Tandem Mass Tag (TMT)11plex Isobaric Label Reagent Set (ThermoFisher Scientific) was utilized for this application. After labeling, equal amounts of sample (peptide) from each liver sample were mixed together. In order to achieve in-depth characterization of the proteome, the labeled peptides were fractionated using 2DLC (basic $\mathrm{pH}$ reverse-phase separation followed by acidic $\mathrm{pH}$ reverse phase) and analyzed on a highresolution, tribrid mass spectrometer (Orbitrap Fusion Tribrid, ThermoFisher Scientific) using conditions optimized at the PRF. MultiNotch MS3 analysis (40) was used to accurately quantify identified proteins/peptides. Data analysis was performed using Proteome Discoverer (v 2.4, ThermoFisher Scientific). MS2 spectra were searched against UniProt mouse protein database (17011 sequences as reviewed entries; downloaded on 05/06/2020) using the following search parameters: MS1 and MS2 tolerance were set to $10 \mathrm{ppm}$ and 0.6 Da, respectively; carbamidomethylation of cysteines (57.02146 Da) and TMT labeling of lysine and N-termini of peptides (229.16293 Da) were considered static modifications; oxidation of methionine (15.9949 Da) and deamidation of asparagine and glutamine (0.98401 Da) were considered variable. Identified proteins and peptides were filtered to retain only those that passed $\leq 2 \%$ false discovery rate (FDR) threshold of detection. Quantitation was performed using high-quality MS3 spectra (Average signal-to-noise ratio of 10 and $<40 \%$ isolation interference). Differential protein expression in each treatment cohort was normalized to the high-fat alone cohort. Protein names retrieved from UniProt.org, and Reactome V78 (reactome.org) was used for pathway enrichment analyses (41) for species Mus musculus. STRING database-v11 (string-db.org) was used to detect protein-protein interactions and additional enrichment analyses provide information related to cellular components, molecular functions, and biological processes by Gene Ontology (GO) annotation. It also offered WikiPathways and Kyoto Encyclopedia of Genes and Genomes (KEGG) databases to curate pathways. Only proteins with a $\leq 2 \%$ FDR confidence of detection were included in the analyses. The individual differential protein expression was established by calculating the 
abundance ratio of normalized abundances of each intervention sample to the high-fat alone samples. For the group analysis, individual data points were merged by groups to obtain means and standard deviations. The initial analysis was an unbiased search of differentially-expressed proteins and significantly altered proteins. Subsequently, we searched the data base, specifically, for changes in the expression of keratins and other differentiation-related proteins. Mass spectrometry-based proteomics data were placed in a data repository known as ProteomeXchange Consortium via the PRIDE partner repository with the dataset identifier PXD030954.

Statistical analysis. Group means and standard deviations were obtained for discrete gross and histochemical features as well as for individual biochemical values (ALT, AST and TG) and individual protein values obtained in the proteomic analysis. Data generated in this way were analyzed by analysis of variance (ANOVA) followed by unpaired t-test (two-tailed) for comparison using GraphPad Prism, version 9. Pathways enrichment data reflect Reactome-generated $p$-values based on the number of entities identified in a given pathway as compared to total proteins responsible for that pathway. The significance ( $p$-value) is calculated by the overrepresentation analysis (hypergeometric distribution). For STRING enrichment analysis, the whole genome statistical background was assumed and FDR stringency was $\leq 1 \%$. Data were considered significant at $p<0.05$. 


\section{RESULTS}

Body weight, liver weight comparisons, liver enzyme levels and triglycerides. Animal weights and liver weights were assessed at euthanasia (Figure 1A). As expected, there were large differences between mice fed the low-fat diet and those on the high-fat diet irrespective of intervention. Inclusion of Aquamin $^{\circledR}$ along with high-fat feeding had no effect on either total body weight or liver weight, but there was a small reduction in both parameters in the cohort of mice receiving OCA as compared to control mice on the high-fat diet. Liver enzyme (ALT and AST) levels and triglyceride levels are shown in Figure 1B. Levels of both ALT and AST increased substantially with high-fat feeding as did triglyceride levels. Both liver enzymes were also increased in mice receiving either Aquamin ${ }^{\circledR}$ or OCA along with high-fat feeding. With OCA, there was a modest reduction compared to levels seen in untreated mice on the same diet. Differences between the interventions and control (high-fat diet-fed mice) were not statistically different for either ALT or AST but all three high-fat groups were different from the low-fat control group. Serum triglyceride levels (evidence of metabolic dysfunction) were significantly elevated with high-fat feeding but neither Aquamin ${ }^{\circledR}$ nor OCA had a significant effect on this parameter.

Histological features. Sections of formalin-fixed liver tissue were stained with hematoxylin and eosin and evaluated for steatosis, ballooning hepatocyte degeneration and inflammation. Additional sections were stained with PSR and assessed for collagen deposition. Data are shown in Figure 2. Steatosis was virtually undetectable in mice on the low-fat control diet but widespread in every animal on the high-fat diet after 16 weeks of feeding. Neither Aquamin ${ }^{\circledR}$ nor OCA reduced steatosis significantly compared to the high-fat control, but both interventions reduced ballooning degeneration and inflammation to some extent. When scores from the three parameters were added together (as in citation 34) to give a NAFLD activity score, both Aquamin ${ }^{\circledR}$ and OCA were different from high-fat alone. However, the degree of elevation was small to begin with (especially with inflammation) and the overall significance of the modest reduction is difficult to gauge. PSR staining demonstrated collagen deposition within the liver parenchyma of mice on the high-fat diet while sections from low-fat control 
mice did not show staining except around vascular elements and in relation to the capsule. Some reduction in the collagen deposition score was noted with both interventions. With Aquamin ${ }^{\circledR}$, the reduction in the collagen deposition reached a level of statistical significance. However, as with inflammation, the overall PSR staining score was low ( $<1.5$ out of 4$)$ after 16 weeks on the high-fat diet. Proteomic analysis: Effects of low-fat diet, Aquamin ${ }^{\circledR}$ and OCA compared to high-fat alone. The number of upregulated and downregulated proteins in C57BL/6 mice on the low-fat diet (relative to high-fat control mice) and the number of up- and downregulated proteins with Aquamin ${ }^{\circledR}$ or OCA in comparison to the high-fat control mice is presented as Figure 3A (2-fold cutoff) and 3B (1.5-fold cutoff). It can be seen from the bar graphs that the low-fat control diet was responsible for a substantial number of protein changes. With the 2-fold cutoff and $\leq 2 \%$ FDR, 182 proteins were upregulated, and 126 proteins were downregulated with the low-fat control diet. With Aquamin ${ }^{\circledR}$ and OCA, a total of 86 and 76 proteins, respectively, were upregulated to the same extent, while only five and eight, respectively, were downregulated. When the less stringent cut-off (1.5-fold) was used, more proteins were altered with each intervention but the trends were the same.

Venn diagrams (Figure $3 \mathrm{~A}$ and $3 \mathrm{~B}$ ) show the overlap between protein alterations with low-fat feeding and the other two interventions. Among the proteins elevated with either Aquamin ${ }^{\circledR}$ or $\mathrm{OCA}$, approximately $50 \%$ (at either cut-off) were in common with proteins upregulated in the low-fat control diet. In contrast, the number of downregulated proteins with either intervention that overlapped with proteins downregulated with low-fat feeding was lower on a percentage basis. This was seen with either cutoff but was more extreme with 2-fold cutoff. Overall, 57 upregulated proteins were common between the two interventions (OCA and Aquamin ${ }^{\circledR}$ ), while only two downregulated proteins were common between these two groups. Supplement Table 2 and Supplement Table 3 provide lists of upregulated and downregulated proteins common at 2-fold among three groups (low-fat, OCA and Aquamin ${ }^{\circledR}$ on high-fat diet) across five mice per group. 
Proteomic analysis: Individual protein changes in response to Aquamin ${ }^{\circledR}$. Figure 4 shows the foldchange distribution of individual proteins responsive to Aquamin ${ }^{\circledR}$ in the presence of high-fat diet. Upregulated proteins that met the criterion of statistical significance at $p<0.05$ are shown in blue while downregulated proteins are shown in red. The bold colors (blue and red) indicate proteins that were statistically different and also at least 1.5 -fold up- or downregulated.

Table $1 \mathrm{~A}$ identifies the upregulated proteins that met the dual criteria (statistical difference from the high-fat control at $p<0.05$ and at least 1.5 -fold increase) in response to Aquamin ${ }^{\circledR}$. Supplement Table 4 provide a comprehensive list of upregulated proteins that met the criterion of statistical difference from control, irrespective of fold-change. While only a small number of proteins (12 in total) met both criteria, a total of 183 upregulated proteins were statistically increased. Reactome pathway analysis was utilized as a way to help delineate how altered protein expression in response to Aquamin ${ }^{\circledR}$ influences biological function. As can be seen from the altered pathways, the upregulated proteins impact lipid metabolism and trafficking of lipids (Table 1B). Supplement Table 5 lists top pathways affected by the altered (upregulated) proteins presented in Supplement Table 4.

Aquamin ${ }^{\circledR}$-responsive proteins the met the dual criteria of 1.5 -fold decrease and statistical significance are shown in Table $1 \mathrm{C}$ and those that were statistically-different (and downregulated) from high-fat control, regardless of fold change, are shown in Supplement Table 6 . A total of 14 proteins met both criteria while a total of 130 were statistically different from the high-fat control, independent of foldchange. As with upregulated proteins, Reactome pathway analysis was used to help identify potential

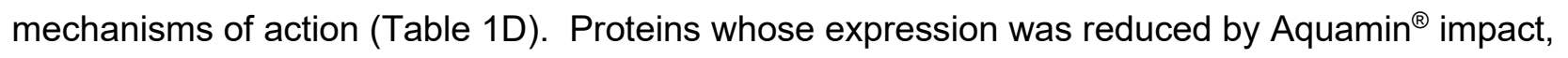
primarily, amino acid metabolism, although purine and pyrimidine metabolism also appear to be targets. Among the downregulated proteins were multiple cytochrome P450 family members (7 in all) including three (i.e., 2C54, 2C50, 2C29) that were among the most highly downregulated. Cytochrome P450 family members are highly-expressed in liver and serve to metabolize and detoxify numerous chemicals, including fats (42). Supplement Table 7 lists top pathways affected by all of the significantly 
downregulated proteins presented in Supplement Table 6. Amino acid metabolism, bile acid and bile salt metabolism and recycling of bile acids and salts are among the significantly downregulated pathways identified.

Tables $1 \mathrm{~A}$ and $1 \mathrm{C}$ also show for comparison how the Aquamin ${ }^{\circledR}$-sensitive proteins responded to low-fat feeding and to OCA. It can be seen that while there was overlap between Aquamin ${ }^{\circledR}$ and OCA (as shown in Figure 3) in the many of the highly upregulated proteins, the response to OCA was not as robust as the response to Aquamin ${ }^{\circledR}$ (i.e., fold-change was smaller and only two were statistically different from control). In regard to downregulated moieties, of the 14 Aquamin ${ }^{\circledR}$-sensitive proteins that met the dual criteria, nine were also responsive to OCA by the same criteria. In contrast, there was little overlap between Aquamin ${ }^{\circledR}$-responsiveness and response to the low-fat diet. In fact, many of the protein changes were in the opposite direction (Tables $1 \mathrm{~A}$ and $1 \mathrm{C}$ ).

Supplement Tables 8 and 9 present proteomic enrichment data curated by the STRING database with 183 significantly upregulated proteins and 130 significantly downregulated proteins, respectively, in response to Aquamin ${ }^{\circledR}$. These datasheets provide information related to biological process, molecular function and cellular components along with additional pathways curated by KEGG and Wiki (as part of STRING enrichment analysis).

After analyzing data based on statistical significance, the proteomic database was searched for the most highly up- and downregulated Aquamin ${ }^{\circledR}$-responsive proteins based on fold-change independent of statistical significance. Table 2 presents a list of the most highly upregulated proteins (2-fold or greater) with Aquamin ${ }^{\circledR}$. As a group, epithelial cell keratins were prominent in the protein list. Of the top 22 most highly upregulated proteins, 10 were keratins (Table 2). Also, nine of these keratins were common at 2-fold-change with the low-fat group (Supplement Table 2). Of interest, the major keratins found in the liver - i.e., keratin 8, and 18 (43) - were not altered (up or down) with Aquamin ${ }^{\circledR}$ (Figure 5). Keratins are structural components of epithelial cells and play key roles in differentiation, 
morphogenesis, barrier formation and tissue integrity. They are also important signaling intermediates (44). How each of the keratins identified here functions in the liver, specifically, is not known. The effects of low-fat feeding and the effects of treatment with OCA on these same proteins are included in Table 2 (complete protein list) and Figure 5 (keratins). As can be seen, some of the same keratins were increased with low-fat feeding but the degree of upregulation was less than that observed with Aquamin $^{\circledR}$. While the low-fat diet does not provide the same trace elements as does Aquamin ${ }^{\circledR}, \mathrm{D} 5008$ chow contains a mineral supplement as part of its formulation. Thus, it is not unreasonable to observe some change in the keratin expression profile in livers of mice on the low-fat diet. In contrast, with the exception of keratin 79 , upregulation of individual keratins with OCA was almost non-existent.

In addition to keratins, three members of the tubulin family were also upregulated in response to Aquamin $^{\circledR}$ (Table 2). Tubulins constitute another set of important structural proteins within cells, as they are the major constituents of the microtubule system of the cytoskeleton (45). Of interest, the tubulin proteins were responsive to both low-fat feeding and OCA treatment as well as to Aquamin ${ }^{\circledR}$ (Table 2).

Reactome pathway analysis was utilized to help identify the various pathways influenced by the protein changes noted in Table 2 with Aquamin ${ }^{\circledR}$. Not surprisingly, pathways related to differentiation were the most highly affected (Table 3). Among these were keratinization, cornified envelope formation, hemidesmosome assembly, gap junction formation and gap junction assembly. In addition, pathways involved in intracellular trafficking of proteins as well as protein trafficking between the cytoplasm and plasma membrane were significantly influenced by the protein changes occurring in response to Aquamin ${ }^{\circledR}$. Protein trafficking events are well-known to depend on cations in the environment $(46,47)$. Another pathway of interest was hedgehog signaling in the off-state. Signaling through the hedgehog pathway is associated with chronic liver injury $(48,49)$ and hedgehog signaling may have implications in hepatocellular carcinomas. All of the Aquamin ${ }^{\circledR}$-responsive Reactome pathways that met the criterial of having an entities $p$ value of $<0.05$ are presented in Table 3 . 
The database was also searched for proteins that were downregulated by 2-fold or greater with Aquamin $^{\circledR}$. A total of five individual proteins met this criterion. Of the proteins in the list, four of the five were also statistically significant and presented as part of Table 1.

Proteomic changes in response to low-fat feeding and to OCA. While the response to Aquamin ${ }^{\circledR}$ was the focus of this study, we also assessed protein changes driven by low-fat feeding or by OCA in highfat mice. Supplement Tables 10 and 11 show proteins upregulated and downregulated, respectively, by 2 -fold or greater with low-fat feeding. Reactome pathways influenced by these protein changes are shown in Supplement Table 12 and 13. A large majority of the protein changes observed with low-fat feeding (both up- and downregulated) are related to fat and carbohydrate metabolism. This is consistent with previous proteomic assessment of diet-influenced protein profile in livers of both rats (50) and mice (51).

Supplement Tables 14 and 15 show protein changes in mice on high-fat diet treated with OCA. Supplement Tables 16 and 17 show pathways responsive to OCA. The most interesting (if not entirely unexpected) finding was the prominent downregulation of pathways related to cholesterol metabolism and bile acid formation. This finding provides a sharp distinction between the likely mechanistic events driven by Aquamin ${ }^{\circledR}$ and those responsive to OCA. This finding helps validate the utility of the proteomic screening approach employed here. 


\section{DISCUSSION}

A wide range of studies (9-11) has demonstrated the susceptibility of rodents to diet-induced NAFLD. Steatosis develops rapidly (within 4-8 weeks) in rats and mice placed on a high-fat or high-fat and -sugar diet. In contrast, end-stage liver disease - i.e., fibrosis/cirrhosis and tumor formation - does not manifest until much later. Tumor formation has been reported to occur as early as after 36 weeks of feeding, but typically 12 or more months is required for widespread neoplastic disease to be seen (911). In our own previous studies $(19,20)$, tumors were widespread in male C57BL/6 mice fed a high-fat diet after 12-18 months but almost non-existent in a cohort of mice euthanized at the 5-month timepoint. Extensive inflammation, parenchymal necrosis and fibrotic nodules were also seen at the later timepoints. Most importantly, our studies demonstrated that providing an adequate supply of dietary minerals along with high-fat feeding substantially reduced the incidence and severity of liver damage. A reduction in tumor formation was especially prominent.

Even though end-stage liver injury in rodents is fully manifested only after an extended feeding period, the cellular and molecular events that bring about damage undoubtedly begin to occur much earlier. It is also likely that interventions that mitigate long-term injury have effects that can be detected much earlier. With this in mind, the present study was carried out to identify hepatic alterations detectable in mice after 16 weeks on a high-fat and -sugar diet compared to control mice on a low-fat diet. As a major part of this study, mineral supplementation along with a different intervention (OCA) with a, presumably, different mechanisms of action were also separately evaluated.

In comparison with low-fat-fed control mice, livers were substantially enlarged in high-fat mice. However, there was no gross evidence of more serious liver damage (i.e., fibrotic nodules or tumors) at the 16-week time-point. At the histological level, steatosis was widespread in virtually every mouse on the high-fat diet (not observed in low-fat controls). Ballooning hepatocyte degeneration was also prominent, but inflammation and collagen deposition were minimal. These effects were accompanied 
by an increase in serum triglycerides and an increase in serum ALT and AST levels. Consistent with previous findings in both rat (50) and mouse (51) models, proteomic assessment demonstrated substantial differences in the protein expression profile between control (low-fat) animals and those on the high-fat diet. The most dramatic differences were in proteins that regulate fat and carbohydrate metabolism. Increases in both lipid storage and lipid oxidation [breakdown] were seen with high-fat feeding, but moieties that regulate protein and nucleic acid turnover were also diet-sensitive. Proteins involved in cholesterol metabolism and bile acid formation were especially sensitive to the high-fat diet. Together, these findings demonstrate that extensive metabolic dysregulation occurs early in response to high-fat-feeding - in parallel with widespread steatosis but while other structural changes in the liver are minimal. The metabolic changes described here should be seen as a "normal" liver's attempt to cope with excessive fat and sugar consumption rather than as evidence of impending liver failure in MS-NASH mouse model. Even the rise in ALT and AST levels noted here in MS-NASH mice (reaching 300-500 U/mL), while substantial, are not necessarily evidence of serious liver damage. Acute chemical-induced liver toxicity in mice is associated with ALT / AST levels in the range of 3000-6000 $\mathrm{U} / \mathrm{mL}(12)$.

In contrast to the substantial differences distinguishing low-fat and high-fat feeding, mineral supplementation - along with the high-fat diet - had no effect on whole body weight, gross liver weight or steatosis. Likewise, there was no improvement in serum triglycerides and no decrease in AST and ALT values as compared to what was seen in high-fat control mice. This is consistent with findings reported earlier that mineral supplementation does not prevent steatosis from developing $(19,20)$ and points to downstream events as targets of Aquamin ${ }^{\circledR}$.

The protein profile observed in response to intervention with Aquamin ${ }^{\circledR}$ supports this view. While a select few proteins involved directly in fat metabolism / lipid transport were modulated with Aquamin $^{\circledR}$, the most striking Aquamin ${ }^{\circledR}$-induced protein changes relate to decreased amino acid metabolism as well as a reduction in purine and pyrimidine catabolism. Also prominent was the 
increased expression of moieties that are part of the epithelial cell differentiation process. Reactome pathway analysis identified keratinization, cornified envelope formation, hemidesmosome formation, junction formation and gap junction assembly as significantly altered pathways. Proteins involved in microtubule assembly were also upregulated in response to Aquamin ${ }^{\circledR}$ and several pathways related to protein trafficking within the cell or between interior and cell surface were identified.

How the protein changes observed in response to Aquamin ${ }^{\circledR}$ in the current study and the reduction in liver tumor formation seen previously in our earlier long-term studies $(19,20)$ are related is not fully understood. In the long-term studies with C57BL/6 mice on a HFWD, formation of multiple neoplastic lesions including aberrant foci, both non-regenerative and regenerative hyperplastic nodules, hepatic adenomas and hepatocellular carcinomas were seen. The majority of the tumors occurred in male mice though females were not completely protected. Mice fed a healthy rodent chow diet had many fewer tumors, but even these were not completely protected after 18 months. Hepatic tumor formation in the context of a high-fat diet and steatosis has been extensively studied and multiple contributing mechanisms are thought to be involved $(3,11,16,17)$. Perhaps among them, the most important is lipotoxicity-driven oxidative stress. Oxidant burden, leading to a highly "mutagenic environment" concurrent with high cell turn over resulting from repeated cycles of injury and repair, is a fertile ground for tumor initiation and progression. The formation of toxic and carcinogenic bile acids (a manifestation of lipid over-feeding) may also contribute to tumor formation. The present study identified Aquamin ${ }^{\circledR}$-sensitive protein changes that suggest reduced catabolism of fats (as well as reduced amino acid, purine and pyrimidine metabolism); all of which could be expected to lower oxidant burden. The reduction in multiple cytochrome P450 enzymes is supportive. The cytochrome P450 enzymes are strongly expressed in liver and rise as part of the organ's response to a toxic environment. Of interest, previous studies have demonstrated that strontium (52), selenium (53) and members of the lanthanide family of rare earth elements (54) - all components of Aquamin ${ }^{\circledR}$ - protect the liver against oxidative 
stress. While liver tumor formation, per se, was not addressed in these earlier studies, reduced oxidative stress and reduced tumor formation are, certainly, consistent.

Aquamin $^{\circledR}$-induced epithelial cell differentiation provides another potential mechanism to counteract cancer-promotion. A wide range of empirical information supports this view.

Epidemiological studies have clearly demonstrated the inverse relationship between differentiation in epithelial cells and tumor incidence in many tissues including those of the gastrointestinal tract (55-59). Animal studies have confirmed this relationship $(60,29,30)$ and cell culture studies have provided mechanistic insight into the inverse relationship between differentiation and reduced tumor formation $(61,62)$. In addition to a reduction in tumor incidence, the expression of differentiation features (assessed histologically) and improved prognosis is correlated with both pre-malignant and malignant epithelial cell tumors (63-67). Calcium, which is the quintessential "driver" of differentiation in epithelial cells (68), is the most abundant mineral in Aquamin ${ }^{\circledR}$. Thus, epithelial cell differentiation induced by calcium in the multi-mineral product may underlie liver tumor suppression by Aquamin ${ }^{\circledR}$. It is welldocumented, furthermore, that several of the trace elements in the multi-mineral product can act like calcimimetic agonists, promoting a "left-shift" in the calcium dose-response curve. A higher affinity for the extracellular calcium-sensing receptor than calcium itself has been documented with several of the cationic trace elements in Aquamin ${ }^{\circledR}(29,69-71)$. Similarly, extracellular calcium-sensing receptor has also been expressed in rat hepatocytes (72). Also consistent with this idea is the fact that keratin upregulation was also seen in low-fat mice, though the degree of induction was lower than that observed with Aquamin ${ }^{\circledR}$ itself.

In both Aquamin ${ }^{\circledR}$-treated animals and low-fat fed mice, the strong keratin upregulation reflected a response in a subset of animals. Why only some animals demonstrated this protein signature is not known, but the finding raises an interesting question: Does the variability in response from animal-toanimal help explain why virtually every mouse fed a high-fat diet rapidly develops steatosis but only a subset progresses to more serious disease? This question cannot be addressed with the presently 
available data but we have recently initiated a large linear study in which liver biopsies are obtained from mice on a high-fat diet after 16-weeks on diet (as in the present study) following which the animals are maintained for a total of 18-months in order to determine if there is an inverse correlation between keratin expression at the early time-point and tumor formation later.

Although the focus of this study was on mineral supplementation, we also included OCA as a separate intervention. OCA has been shown to inhibit features of NAFLD / NASH in human patients (21-24) and in mice (25). In the current study, OCA produced a modest reduction in steatosis (histology) and a corresponding reduction in serum triglyceride, AST and ALT levels. In the proteomic screen, there was some overlap in upregulated proteins between Aquamin ${ }^{\circledR}$ and OCA, but OCA did not have the same effect on epithelial differentiation proteins as was seen with Aquamin ${ }^{\circledR}$. In regard to downregulated proteins, the changes seen with OCA were related to cholesterol metabolism and bile acid synthesis, consistent with OCA's function as a Farnesoid X Receptor agonist (21-24). Given these observations, it is unlikely that the two agents - Aquamin ${ }^{\circledR}$ and OCA - would overlap mechanistically in regard to tumor suppression in a meaningful way. Whether they might produce synergistic anti-tumor activity is not known but is a question worth addressing.

NAFLD is a rising public health challenge. Currently, public health measures focus on "lifestyle" changes, especially diet, to prevent the development of steatosis. Such an approach will not work with everyone. Perhaps efforts directed at reducing the downstream consequences of fatty liver infiltration would be of value. It should be noted in this regard that a majority of individuals in Western society do not meet USDA daily intake guidelines for calcium (73). Many individuals are also deficient in magnesium (74) and, presumably, in other minerals that are nutritionally associated with calcium and magnesium. Inadequate mineral intake is not limited to individuals consuming a western style diet. A recent study demonstrated that a majority of individuals in many developing regions of the world also lacked an adequate amount of calcium in their diet $(75,76)$. Whether the mineral supplement used here or some other formulation might provide a way to insure adequate mineral intake remains to be seen. 
We have recently completed a 90-day pilot phase trial in which 30 healthy human subjects were randomized to receive Aquamin ${ }^{\circledR}$ formulated to provide $800 \mathrm{mg}$ of calcium per day, calcium carbonate at the same level or placebo $(37,77)$. To summarize, no safety or tolerability issues were seen with Aquamin $^{\circledR}$. At the same time, colon biopsies obtained before and after treatment demonstrated upregulation of several differentiation-related proteins in the colonic mucosa. In the calcium-alone group, differentiation proteins were also induced, but the levels of increase were lower than seen with Aquamin ${ }^{\circledR}$. Finally, a decrease in the levels of certain primary and secondary bile acids was also observed in subjects receiving Aquamin ${ }^{\circledR}$ in conjunction with altered gut microbial profile. These metabolomic and microbial changes were not observed with calcium alone. While the focus of these clinical studies has been colonic health, the same approach may provide benefit in the liver.

In summary, our past studies have clearly demonstrated the importance of adequate mineral intake for preventing the downstream consequences of fatty liver accumulation in a murine model. The present studies provide mechanistic insight into how mineral supplementation may contribute to reduction in liver tumor formation, one of the most devastating consequences of fatty liver disease in the face of steatosis.

\section{DATA AVAILABILITY STATEMENT}

All relevant data are within the manuscript and its Supporting Information files. The mass spectrometry proteomics data are available on ProteomeXchange Consortium (PRIDE partner repository) - dataset identifier PXD030954.

\section{ETHICS STATEMENT}

For this murine study we utilized NASH mouse model known as MS-NASH. The entire in-life portion of the study and euthanasia was carried out by Crown Bio, Inc., at their Lafayette, LA (USA) 
facility under the approved Standard Operating Procedures at the testing site. All procedures involving live animals were reviewed and approved by the Institutional Animal Care and Use Committee (IACUC). Crown Bio, Inc., is an American Association for Accreditation of Laboratory Animal Care (AAALAC)-accredited institution.

\section{AUTHOR CONTRIBUTIONS}

All the listed authors have contributed toward this project to attain authorship status according to ICMJE criteria. Their specific contributions are as follows; Conceptualization: MNA, JV; Methodology, MNA, JV; Software, MNA; Validation, MNA, SDM, RNK, JV; Formal Analysis, MNA, SDM, RNK, IH, DZ, MAHJ-M; Investigation, MNA, SDM, RNK, IH, DZ, MAHJ-M, JV; Resources, MNA, JV; Data Curation, MNA, SDM, RNK, IH, DZ, MAHJ-M; Writing-Original Draft Preparation, MNA, JV; Writing-Review \& Editing, MNA, SDM, RNK, IH, DZ, MAHJ-M, JV; Visualization, MNA; Supervision, MNA, JV; Project Administration, MNA, JV; Funding Acquisition, JV. All authors have read and agreed to this version of the manuscript.

\section{FUNDING}

This study was funded by NIH grant CA201782 including supplemental funding through the Office of Dietary Supplements (to JV).

\section{ACKNOWLEDGMENTS}

We thank Marigot LTD (Cork, Ireland) for providing Aquamin ${ }^{\circledR}$ Soluble as a gift. Marigot LTD had no role or influence in study design, data collection and analysis, decision to publish, or preparation of the manuscript. We also thank the Proteomics Resource Facility (Pathology Department) for assistance with proteomic data acquisition. 


\section{SUPPLEMENTARY MATERIAL}

The following files are available as part of supplemental information.

S Table 1: Mineral Composition of Aquamin ${ }^{\circledR}$ Soluble; S Table 2: Common and unique upregulated proteins (at 2-fold change threshold); S Table 3: Common and unique downregulated proteins (at 2fold change threshold); S Table 4: Comprehensive list of upregulated (and significantly altered) proteins with Aquamin in high-fat mice; S Table 5: Top pathways associated with significantly upregulated proteins (shown in S Table 4) altered with Aquamin; S Table 6: Comprehensive list of downregulated (and significantly altered) proteins with Aquamin in high-fat mice; S Table 7: Top pathways associated with significantly downregulated proteins (shown in S Table 5) altered with Aquamin; S Table 8: String enrichment analysis of upregulated proteins (presented in S Table 4); S Table 9: String enrichment analysis of downregulated proteins (presented in S Table 6); S Table 10: Upregulated Proteins by an unbiased proteomic screening of C57BL6 mice on low-fat diet; S Table 11: Downregulated Proteins by an unbiased proteomic screening of C57BL6 mice on low-fat diet; S Table 12: Top pathways associated with upregulated proteins altered with low-fat diet in C57BL6 mice; S Table 13: Top pathways associated with downregulated proteins altered with low-fat diet in C57BL6 mice; S Table 14: Upregulated Proteins by an unbiased proteomic screening with Obeticholic acid (OCA) in high-fat mice;

S Table 15: Downregulated Proteins by an unbiased proteomic screening with Obeticholic acid (OCA) in high-fat mice; S Table 16: Top pathways associated with upregulated proteins altered with Obeticholic acid (OCA); S Table 17: Top pathways associated with downregulated proteins altered with Obeticholic acid (OCA)

\section{CONFLICT OF INTEREST}

The authors declare that the research activities were conducted in the absence of any commercial or financial relationships that could be construed as a potential conflict of interest. There is no conflict of interest to declare. 


\section{REFERENCES}

1. Younossi, Z., Anstee, Q. M., Marietti, M., Hardy, T., Henry, L., Eslam, M. et al. (2018). Global burden of NAFLD and NASH: trends, predictions, risk factors and prevention. Nature reviews. Gastroenterology \& hepatology, 15(1), 11-20. https://doi.org/10.1038/nrgastro.2017.109

2. Younossi, Z. M., Koenig, A. B., Abdelatif, D., Fazel, Y., Henry, L., \& Wymer, M. (2016). Global epidemiology of nonalcoholic fatty liver disease-Meta-analytic assessment of prevalence, incidence, and outcomes. Hepatology (Baltimore, Md.), 64(1), 73-84. https://doi.org/10.1002/hep.28431

3. Anstee, Q. M., Reeves, H. L., Kotsiliti, E., Govaere, O., \& Heikenwalder, M. (2019). From NASH to HCC: current concepts and future challenges. Nature reviews. Gastroenterology \& hepatology, 16(7), 411-428. https://doi.org/10.1038/s41575-019-0145-7

4. Cotter, T. G., \& Rinella, M. (2020). Nonalcoholic Fatty Liver Disease 2020: The State of the Disease. Gastroenterology, 158(7), 1851-1864. https://doi.org/10.1053/i.gastro.2020.01.052

5. Sheka, A. C., Adeyi, O., Thompson, J., Hameed, B., Crawford, P. A., \& Ikramuddin, S. (2020). Nonalcoholic Steatohepatitis: A Review. JAMA, 323(12), 1175-1183. https://doi.org/10.1001/jama.2020.2298

6. Hardy, T., Oakley, F., Anstee, Q. M., \& Day, C. P. (2016). Nonalcoholic Fatty Liver Disease: Pathogenesis and Disease Spectrum. Annual review of pathology, 11, 451-496. https://doi.org/10.1146/annurev-pathol-012615-044224

7. Day, C. P., \& James, O. F. (1998). Steatohepatitis: a tale of two "hits"? Gastroenterology, 114(4), 842-845. https://doi.org/10.1016/s0016-5085(98)70599-2

8. Day, C. P., \& Saksena, S. (2002). Non-alcoholic steatohepatitis: definitions and pathogenesis. Journal of gastroenterology and hepatology, 17 Suppl 3, S377-S384. https://doi.org/10.1046/j.1440-1746.17.s3.31.x

9. Nevzorova, Y. A., Boyer-Diaz, Z., Cubero, F. J., \& Gracia-Sancho, J. (2020). Animal models for liver disease - A practical approach for translational research. Journal of hepatology, 73(2), 423-440. https://doi.org/10.1016/j.jhep.2020.04.011

10. Clapper, J. R., Hendricks, M. D., Gu, G., Wittmer, C., Dolman, C. S., Herich, J., et al. (2013). Diet-induced mouse model of fatty liver disease and nonalcoholic steatohepatitis reflecting clinical disease progression and methods of assessment. American journal of physiology. Gastrointestinal and liver physiology, 305(7), G483-G495. https://doi.org/10.1152/ajpgi.00079.2013

11. Febbraio, M. A., Reibe, S., Shalapour, S., Ooi, G. J., Watt, M. J., \& Karin, M. (2019). Preclinical Models for Studying NASH-Driven HCC: How Useful Are They? Cell metabolism, 29(1), 18-26. https://doi.org/10.1016/i.cmet.2018.10.012 
12. Zhang, G., Wang, X., Chung, T. Y., Ye, W., Hodge, L., Zhang, L., et al. (2020). Carbon tetrachloride $\left(\mathrm{CCl}_{4}\right)$ accelerated development of non-alcoholic fatty liver disease (NAFLD)/steatohepatitis (NASH) in MS-NASH mice fed western diet supplemented with fructose (WDF). BMC gastroenterology, 20(1), 339. https://doi.org/10.1186/s12876-020-01467-w

13. Brol, M. J., Rösch, F., Schierwagen, R., Magdaleno, F., Uschner, F. E., Manekeller, S. et al. (2019). Combination of $\mathrm{CCl}_{4}$ with alcoholic and metabolic injuries mimics human liver fibrosis. American journal of physiology. Gastrointestinal and liver physiology, 317(2), G182G194. https://doi.org/10.1152/ajpgi.00361.2018

14. Honda, H., Ikejima, K., Hirose, M., Yoshikawa, M., Lang, T., Enomoto, N. et al. (2002). Leptin is required for fibrogenic responses induced by thioacetamide in the murine liver. Hepatology (Baltimore, Md.), 36(1), 12-21. https://doi.org/10.1053/ihep.2002.33684

15. Marcolin, E., Forgiarini, L. F., Tieppo, J., Dias, A. S., Freitas, L. A., \& Marroni, N. P. (2011). Methionine- and choline-deficient diet induces hepatic changes characteristic of non-alcoholic steatohepatitis. Arquivos de gastroenterologia, 48(1), 72-79. https://doi.org/10.1590/s0004$\underline{28032011000100015}$

16. Zaki, M., Mahdi, A. K., Patman, G. L., Whitehead, A., Maurício, J. P., McCain, M. V. et al. (2021). Key features of the environment promoting liver cancer in the absence of cirrhosis. Scientific reports, 11(1), 16727. https://doi.org/10.1038/s41598-021-96076-2

17. Dowman, J. K., Hopkins, L. J., Reynolds, G. M., Nikolaou, N., Armstrong, M. J., Shaw, J. C. et al. (2014). Development of hepatocellular carcinoma in a murine model of nonalcoholic steatohepatitis induced by use of a high-fat/fructose diet and sedentary lifestyle. The American journal of pathology, 184(5), 1550-1561. https://doi.org/10.1016/j.ajpath.2014.01.034

18. Tetri, L. H., Basaranoglu, M., Brunt, E. M., Yerian, L. M., \& Neuschwander-Tetri, B. A. (2008). Severe NAFLD with hepatic necroinflammatory changes in mice fed trans fats and a highfructose corn syrup equivalent. American journal of physiology. Gastrointestinal and liver physiology, 295(5), G987-G995. https://doi.org/10.1152/ajpgi.90272.2008

19. Nadeem Aslam, M., Bassis, C. M., Zhang, L., Zaidi, S., Varani, J., \& Bergin, I. L. (2016). Calcium Reduces Liver Injury in Mice on a High-Fat Diet: Alterations in Microbial and Bile Acid Profiles. PloS one, 11(11), e0166178. https://doi.org/10.1371/journal.pone.0166178

20. Aslam, M. N., Bergin, I., Naik, M., Hampton, A., Allen, R., Kunkel, S. L. et al. (2012). A multimineral natural product inhibits liver tumor formation in C57BL/6 mice. Biological trace element research, 147(1-3), 267-274. https://doi.org/10.1007/s12011-011-9316-2

21. Sepe, V., Distrutti, E., Fiorucci, S., \& Zampella, A. (2018). Farnesoid X receptor modulators 2014-present: a patent review. Expert opinion on therapeutic patents, 28(5), 351-364. https://doi.org/10.1080/13543776.2018.1459569 
22. Neuschwander-Tetri B. A. (2012). Farnesoid x receptor agonists: what they are and how they might be used in treating liver disease. Current gastroenterology reports, 14(1), 55-62. https://doi.org/10.1007/s11894-011-0232-6

23. Mudaliar, S., Henry, R. R., Sanyal, A. J., Morrow, L., Marschall, H. U., Kipnes, M. et al. (2013). Efficacy and safety of the farnesoid $X$ receptor agonist obeticholic acid in patients with type 2 diabetes and nonalcoholic fatty liver disease. Gastroenterology, 145(3), 574-82.e1. https://doi.org/10.1053/j.gastro.2013.05.042

24. Neuschwander-Tetri, B. A., Loomba, R., Sanyal, A. J., Lavine, J. E., Van Natta, M. L., Abdelmalek, M. F. et al. (2015). Farnesoid X nuclear receptor ligand obeticholic acid for noncirrhotic, non-alcoholic steatohepatitis (FLINT): a multicentre, randomised, placebo-controlled trial. Lancet (London, England), 385(9972), 956-965. https://doi.org/10.1016/S0140$\underline{6736(14) 61933-4}$

25. Haczeyni, F., Poekes, L., Wang, H., Mridha, A. R., Barn, V., Geoffrey Haigh, W. et al. (2017). Obeticholic acid improves adipose morphometry and inflammation and reduces steatosis in dietary but not metabolic obesity in mice. Obesity (Silver Spring, Md.), 25(1), 155-165. https://doi.org/10.1002/oby.21701

26. Adey, W., McKibbin, D. (1970). Studies on the Maerl Species Phymatolithon calcareum (Pallas) nov. comb. and Lithothamnium coralloides Crouan in the Ria de Vigo., 13(2), 100-106. doi:10.1515/botm.1970.13.2.100.

27. Ward, D. T., \& Riccardi, D. (2012). New concepts in calcium-sensing receptor pharmacology and signalling. British journal of pharmacology, 165(1), 35-48. https://doi.org/10.1111/j.14765381.2011.01511.x

28. Pi, M., Faber, P., Ekema, G., Jackson, P. D., Ting, A., Wang, N., et al. (2005). Identification of a novel extracellular cation-sensing G-protein-coupled receptor. The Journal of biological chemistry, 280(48), 40201-40209. https://doi.org/10.1074/jbc.M505186200

29. Attili, D., Jenkins, B., Aslam, M. N., Dame, M. K., \& Varani, J. (2012). Growth control in colon epithelial cells: gadolinium enhances calcium-mediated growth regulation. Biological trace element research, 150(1-3), 467-476. https://doi.org/10.1007/s12011-012-9503-9

30. Aslam, M. N., Paruchuri, T., Bhagavathula, N., \& Varani, J. (2010). A mineral-rich red algae extract inhibits polyp formation and inflammation in the gastrointestinal tract of mice on a highfat diet. Integrative cancer therapies, 9(1), 93-99. https://doi.org/10.1177/1534735409360360

31. Aslam, M. N., Bergin, I., Naik, M., Paruchuri, T., Hampton, A., Rehman, M., et al. (2012). A multimineral natural product from red marine algae reduces colon polyp formation in C57BL/6 mice. Nutrition and cancer, 64(7), 1020-1028. https://doi.org/10.1080/01635581.2012.713160 
32. Sun, G., Jackson, C. V., Zimmerman, K., Zhang, L. K., Finnearty, C. M., Sandusky, G. E., et al. (2019). The FATZO mouse, a next generation model of type 2 diabetes, develops NAFLD and NASH when fed a Western diet supplemented with fructose. BMC gastroenterology, 19(1), 41. https://doi.org/10.1186/s12876-019-0958-4

33. Neff E. P. (2019). Farewell, FATZO: a NASH mouse update. Lab animal, 48(6), 151. https://doi.org/10.1038/s41684-019-0311-0

34. Kleiner, D. E., Brunt, E. M., Van Natta, M., Behling, C., Contos, M. J., Cummings, O. W. et al. (2005). Design and validation of a histological scoring system for nonalcoholic fatty liver disease. Hepatology (Baltimore, Md.), 41(6), 1313-1321. https://doi.org/10.1002/hep.20701

35. Pais, P., \& D'Amato, M. (2014). In vivo efficacy study of milk thistle extract (ETHIS-094 ${ }^{\text {TM }}$ ) in STAM $^{\mathrm{TM}}$ model of nonalcoholic steatohepatitis. Drugs in R\&D, 14(4), 291-299. https://doi.org/10.1007/s40268-014-0068-2

36. Piguet, A. C., Saran, U., Simillion, C., Keller, I., Terracciano, L., Reeves, H. L., et al. (2015). Regular exercise decreases liver tumors development in hepatocyte-specific PTEN-deficient mice independently of steatosis. Journal of hepatology, 62(6), 1296-1303. https://doi.org/10.1016/j.jhep.2015.01.017

37. Aslam, M. N., McClintock, S. D., Jawad-Makki, M., Knuver, K., Ahmad, H. M., Basrur, V., et al. (2021). A Multi-Mineral Intervention to Modulate Colonic Mucosal Protein Profile: Results from a 90-Day Trial in Human Subjects. Nutrients, 13(3), 939. https://doi.org/10.3390/nu13030939

38. Attili, D., McClintock, S. D., Rizvi, A. H., Pandya, S., Rehman, H., Nadeem, D. M., et al. (2019). Calcium-induced differentiation in normal human colonoid cultures: Cell-cell / cell-matrix adhesion, barrier formation and tissue integrity. PloS one, 14(4), e0215122. https://doi.org/10.1371/journal.pone.0215122

39. Aslam, M. N., McClintock, S. D., Attili, D., Pandya, S., Rehman, H., Nadeem, D. M., et al. (2020). Ulcerative Colitis-Derived Colonoid Culture: A Multi-Mineral-Approach to Improve Barrier Protein Expression. Frontiers in cell and developmental biology, 8, 577221. https://doi.org/10.3389/fcell.2020.577221

40. McAlister, G. C., Nusinow, D. P., Jedrychowski, M. P., Wühr, M., Huttlin, E. L., Erickson, B. K. et al. (2014). MultiNotch MS3 enables accurate, sensitive, and multiplexed detection of differential expression across cancer cell line proteomes. Analytical chemistry, 86(14), 7150-7158. https://doi.org/10.1021/ac502040v

41. Fabregat, A., Sidiropoulos, K., Garapati, P., Gillespie, M., Hausmann, K., Haw, R., et al. (2016). The Reactome pathway Knowledgebase. Nucleic acids research, 44(D1), D481-D487. https://doi.org/10.1093/nar/gkv1351

42. McDonnell, A. M., \& Dang, C. H. (2013). Basic review of the cytochrome p450 system. Journal of the advanced practitioner in oncology, 4(4), 263-268.

https://doi.org/10.6004/jadpro.2013.4.4.7 
43. Golob-Schwarzl, N., Bettermann, K., Mehta, A. K., Kessler, S. M., Unterluggauer, J., Krassnig, S., et al. (2019). High Keratin 8/18 Ratio Predicts Aggressive Hepatocellular Cancer Phenotype. Translational oncology, 12(2), 256-268. https://doi.org/10.1016/j.tranon.2018.10.010

44. Dmello, C., Srivastava, S. S., Tiwari, R., Chaudhari, P. R., Sawant, S., \& Vaidya, M. M. (2019). Multifaceted role of keratins in epithelial cell differentiation and transformation. Journal of biosciences, 44(2), 33. https://doi.org/10.1007/s12038-019-9864-8

45. Binarová, P., \& Tuszynski, J. (2019). Tubulin: Structure, Functions and Roles in Disease. Cells, 8(10), 1294. https://doi.org/10.3390/cells8101294

46. Graber, Z. T., Shi, Z., \& Baumgart, T. (2017). Cations induce shape remodeling of negatively charged phospholipid membranes. Physical chemistry chemical physics : PCCP, 19(23), 15285-15295. https://doi.org/10.1039/c7cp00718c

47. Tiwari, S., Askari, J. A., Humphries, M. J., \& Bulleid, N. J. (2011). Divalent cations regulate the folding and activation status of integrins during their intracellular trafficking. Journal of cell science, 124(Pt 10), 1672-1680. https://doi.org/10.1242/jcs.084483

48. Shen, X., Peng, Y., \& Li, H. (2017). The Injury-Related Activation of Hedgehog Signaling Pathway Modulates the Repair-Associated Inflammation in Liver Fibrosis. Frontiers in immunology, 8, 1450. https://doi.org/10.3389/fimmu.2017.01450

49. Huang, S., He, J., Zhang, X., Bian, Y., Yang, L., Xie, G., et al. (2006). Activation of the hedgehog pathway in human hepatocellular carcinomas. Carcinogenesis, 27(7), 1334-1340. https://doi.org/10.1093/carcin/bgi378

50. Sang, J., Qu, H., Gu, R., Chen, D., Chen, X., Yin, B., et al. (2019). Proteomics study of the effect of high-fat diet on rat liver. The British journal of nutrition, 122(9), 1062-1072. https://doi.org/10.1017/S0007114519001740

51. Khan, Z. N., Leite, A., Charone, S., Sabino, I. T., Martini, T., Pereira, H. A., et al. (2016). Liver proteome of mice with different genetic susceptibilities to the effects of fluoride. Journal of applied oral science : revista FOB, 24(3), 250-257. https://doi.org/10.1590/1678-775720150364

52. Jiang, H., Guan, Q., Xiao, Y., Feng, Z., Yu, G., \& Pan, Q. (2018). Strontium Alleviates Endoplasmic Reticulum Stress in a Nonalcoholic Fatty Liver Disease Model. Journal of medicinal food, 10.1089/jmf.2018.4186. https://doi.org/10.1089/jmf.2018.4186

53. Miyata, M., Matsushita, K., Shindo, R., Shimokawa, Y., Sugiura, Y., \& Yamashita, M. (2020). Selenoneine Ameliorates Hepatocellular Injury and Hepatic Steatosis in a Mouse Model of NAFLD. Nutrients, 12(6), 1898. https://doi.org/10.3390/nu12061898

54. Li, R., Yu, L., Qin, Y., Zhou, Y., Liu, W., Li, Y., et al. (2021). Protective effects of rare earth lanthanum on acute ethanol-induced oxidative stress in mice via Keap 1/Nrf2/p62 
activation. The Science of the total environment, 758, 143626.

https://doi.org/10.1016/j.scitotenv.2020.143626

55. Keum, N., Aune, D., Greenwood, D. C., Ju, W., \& Giovannucci, E. L. (2014). Calcium intake and colorectal cancer risk: dose-response meta-analysis of prospective observational studies. International journal of cancer, 135(8), 1940-1948. https://doi.org/10.1002/ijc.28840

56. Kesse, E., Boutron-Ruault, M. C., Norat, T., Riboli, E., Clavel-Chapelon, F., \& E3N Group (2005). Dietary calcium, phosphorus, vitamin D, dairy products and the risk of colorectal adenoma and cancer among French women of the E3N-EPIC prospective study. International journal of cancer, 117(1), 137-144. https://doi.org/10.1002/ijc.21148

57. Flood, A., Peters, U., Chatterjee, N., Lacey, J. V., Jr, Schairer, C., \& Schatzkin, A. (2005). Calcium from diet and supplements is associated with reduced risk of colorectal cancer in a prospective cohort of women. Cancer epidemiology, biomarkers \& prevention : a publication of the American Association for Cancer Research, cosponsored by the American Society of Preventive Oncology, 14(1), 126-132

58. Shaukat, A., Scouras, N., \& Schünemann, H. J. (2005). Role of supplemental calcium in the recurrence of colorectal adenomas: a metaanalysis of randomized controlled trials. The American journal of gastroenterology, 100(2), 390-394. https://doi.org/10.1111/j.1572$\underline{0241.2005 .41220 . x}$

59. Larsson, S. C., Bergkvist, L., Rutegård, J., Giovannucci, E., \& Wolk, A. (2006). Calcium and dairy food intakes are inversely associated with colorectal cancer risk in the Cohort of Swedish Men. The American journal of clinical nutrition, 83(3), 667-729. https://doi.org/10.1093/ajcn.83.3.667

60. Newmark, H. L., Yang, K., Kurihara, N., Fan, K., Augenlicht, L. H., \& Lipkin, M. (2009). Westernstyle diet-induced colonic tumors and their modulation by calcium and vitamin D in C57BI/6 mice: a preclinical model for human sporadic colon cancer. Carcinogenesis, 30(1), 88-92. https://doi.org/10.1093/carcin/bgn229

61. Mariadason, J. M., Bordonaro, M., Aslam, F., Shi, L., Kuraguchi, M., Velcich, A., et al. (2001). Down-regulation of beta-catenin TCF signaling is linked to colonic epithelial cell differentiation. Cancer research, 61(8), 3465-3471.

62. Chakrabarty, S., Radjendirane, V., Appelman, H., \& Varani, J. (2003). Extracellular calcium and calcium sensing receptor function in human colon carcinomas: promotion of E-cadherin expression and suppression of beta-catenin/TCF activation. Cancer research, 63(1), 67-71.

63. Sievers, C. K., Leystra, A. A., Clipson, L., Dove, W. F., \& Halberg, R. B. (2016). Understanding Intratumoral Heterogeneity: Lessons from the Analysis of At-Risk Tissue and Premalignant Lesions in the Colon. Cancer prevention research (Philadelphia, Pa.), 9(8), 638-641. https://doi.org/10.1158/1940-6207.CAPR-16-0096 
64. Morson, B. C., Whiteway, J. E., Jones, E. A., Macrae, F. A., \& Williams, C. B. (1984). Histopathology and prognosis of malignant colorectal polyps treated by endoscopic polypectomy. Gut, 25(5), 437-444. https://doi.org/10.1136/gut.25.5.437

65. Cunningham, K. N., Mills, L. R., Schuman, B. M., \& Mwakyusa, D. H. (1994). Long-term prognosis of well-differentiated adenocarcinoma in endoscopically removed colorectal adenomas. Digestive diseases and sciences, 39(9), 2034-2037. https://doi.org/10.1007/BF02088143

66. Xu, B., Yu, L., Zhao, L. Z., \& Ma, D. W. (2016). Prognostic factors in the patients with T2N0M0 colorectal cancer. World journal of surgical oncology, 14, 76. https://doi.org/10.1186/s12957$\underline{016-0826-4}$

67. Xiao, H., Yoon, Y. S., Hong, S. M., Roh, S. A., Cho, D. H., Yu, C. S., et al. (2013). Poorly differentiated colorectal cancers: correlation of microsatellite instability with clinicopathologic features and survival. American journal of clinical pathology, 140(3), 341-347. https://doi.org/10.1309/AJCP8P2DYNKGRBVI

68. Stewart, T. A., Yapa, K. T., \& Monteith, G. R. (2015). Altered calcium signaling in cancer cells. Biochimica et biophysica acta, 1848(10 Pt B), 2502-2511. https://doi.org/10.1016/j.bbamem.2014.08.016

69. Takaoka, S., Yamaguchi, T., Yano, S., Yamauchi, M., \& Sugimoto, T. (2010). The Calciumsensing Receptor ( $\mathrm{CaR})$ is involved in strontium ranelate-induced osteoblast differentiation and mineralization. Hormone and metabolic research $=$ Hormon- und Stoffwechselforschung = Hormones et metabolisme, 42(9), 627-631. https://doi.org/10.1055/s-0030-1255091

70. Huang, Y., Zhou, Y., Castiblanco, A., Yang, W., Brown, E. M., \& Yang, J. J. (2009). Multiple $\mathrm{Ca}(2+)$-binding sites in the extracellular domain of the $\mathrm{Ca}(2+)$-sensing receptor corresponding to cooperative $\mathrm{Ca}(2+)$ response. Biochemistry, 48(2), 388-398. https://doi.org/10.1021/bi8014604

71. Carrillo-López, N., Fernández-Martín, J. L., Alvarez-Hernández, D., González-Suárez, I., Castro-Santos, P., Román-García, P., et al. (2010). Lanthanum activates calcium-sensing receptor and enhances sensitivity to calcium. Nephrology, dialysis, transplantation : official publication of the European Dialysis and Transplant Association - European Renal Association, 25(9), 2930-2937. https://doi.org/10.1093/ndt/gfq124

72. Canaff, L., Petit, J. L., Kisiel, M., Watson, P. H., Gascon-Barré, M., \& Hendy, G. N. (2001). Extracellular calcium-sensing receptor is expressed in rat hepatocytes. coupling to intracellular calcium mobilization and stimulation of bile flow. The Journal of biological chemistry, 276(6), 4070-4079. https://doi.org/10.1074/jbc.M009317200

73. U.S. Department of Health and Human Services; U.S. Department of Agriculture. 2015-2020 Dietary Guidelines for Americans. December 2015, 8th ed.; U.S. Department of Health and Human Services: Washington, DC, USA; U.S. Department of Agriculture: Washington, DC, 
USA, 2015. Available online: https://health.gov/our-work/food-and-nutrition/2015-2020-dietaryguidelines/. (accessed on 6 January 2022).

74. Lennie, T. A., Andreae, C., Rayens, M. K., Song, E. K., Dunbar, S. B., Pressler, S. J., et al. (2018). Micronutrient Deficiency Independently Predicts Time to Event in Patients With Heart Failure. Journal of the American Heart Association, 7(17), e007251. https://doi.org/10.1161/JAHA.117.007251

75. Bai, Y., Herforth, A., \& Masters, W. A. (2022). Global variation in the cost of a nutrient-adequate diet by population group: an observational study. The Lancet. Planetary health, 6(1), e19-e28. https://doi.org/10.1016/S2542-5196(21)00285-0

76. Kopru, S., Cadir, M., \& Soylak, M. (2022). Investigation of Trace Elements in Vegan Foods by ICP-MS After Microwave Digestion. Biological trace element research, 10.1007/s12011-02203106-9. https://doi.org/10.1007/s12011-022-03106-9

77. Aslam, M. N., Bassis, C. M., Bergin, I. L., Knuver, K., Zick, S. M., Sen, A. et al. (2020). A Calcium-Rich Multimineral Intervention to Modulate Colonic Microbial Communities and Metabolomic Profiles in Humans: Results from a 90-Day Trial. Cancer prevention research (Philadelphia, Pa.), 13(1), 101-116. https://doi.org/10.1158/1940-6207.CAPR-19-0325 


\section{FIGURE LEGENDS}

Figure 1. Whole body weight, liver weight and biochemical markers. A. Whole body weight and liver weight were obtained for each animal at euthanasia. B. Blood was obtained by cardiac puncture at euthanasia and assessed for ALT, AST and triglyceride levels. Values shown are means and standard deviations based on 9 animals per group. Statistical significance was assessed by ANOVA followed by two-tailed t-test. Asterisks $\left(^{*}\right)$ indicate statistical difference from high-fat control at $p<0.05$. With all 5 parameters, low-fat control values were statistically different from high-fat control at $p<0.05$.

Figure 2. Histological features. H\&E-stained sections were evaluated for steatosis, ballooning hepatocyte degeneration and inflammation by two trained individuals who did not know the treatment group from which the section was obtained. Scoring of each parameter was as described in the Materials and Methods Section and followed the Kleiner system (34). The NAFLD activity score (NAS) is a summation of the three individual parameters (steatosis, ballooning hepatocyte degeneration and inflammation), calculated in each mouse. Picrosirius red (PSR)-stained sections were evaluated for collagen deposition. Scoring of each parameter was as described in the Materials and Methods Section. Values shown are means and standard deviations based on 9 animals per group. Statistical significance was assessed by ANOVA followed by two-tailed t-test. Asterisks $\left({ }^{*}\right)$ indicate statistical difference from high-fat control at $p<0.05$. For steatosis panels, bar $=200 \mu \mathrm{m}$, for ballooning degeneration and PSR panels, bar=50 $\mu \mathrm{m}$ and for inflammation in low-fat, bar=50 $\mu \mathrm{m}$, and in high-fat, bar $=200 \mu \mathrm{m}$. For inflammation in high-fat, a small panel is showing an inflammatory lesion at a higher resolution, bar $=50 \mu \mathrm{m}$.

Figure 3. Proteomic analysis. Lysates were prepared from liver tissue of individual mice (5 per group) and subjected to Tandem Mass Tag (TMT) labeling / mass spectroscopy as described in the Materials and Methods Section. Data from individual animals were merged by treatment group for analysis.

Left panels: Abundance ratios of all proteins were calculated by comparing the normalized abundance 
of each group to the high-fat control group. The upper panel indicates the number of proteins that was increased or decreased by 2 -fold $(\mathbf{A})$ or greater (with $\leq 2 \%$ FDR) and the lower panel indicates the number of proteins that was increased or decreased by 1.5 -fold (B) or greater (with $\leq 2 \%$ FDR). Right panels: Venn diagrams showing the overlap in the number of proteins altered (increased or decreased) by an average of 2-fold (A) or greater (upper panel) or 1.5-fold (B) or greater (lower panel) among the three treatment groups relative to the high-fat control and unique to each group.

Figure 4. Distribution of proteins upregulated or downregulated in response to Aquamin ${ }^{\circledR}$ compared to high-fat control. Values for each protein (group average) in the high-fat control was set to 1.0 and values in the Aquamin ${ }^{\circledR}$ group compared to this. The $\mathrm{x}$-axis shows the fold-change (log10) of individual proteins and the $y$-axis reflects individual protein $p$-values ( $\mathrm{N}=5$ individual liver samples per group). The mass of proteins shown in black represent proteins that were less than 1.5-fold different from control (up or down) and not statistically significant. Proteins depicted with gray were different from control by 1.5 -fold or greater but not statistically different. Red triangles represent down-regulated proteins and blue triangles represent up-regulated proteins that were statistically significant as compared to control. Bold color (red or blue) represents proteins that were both statistically different from control and different from control by 1.5 -fold or greater. Certain individual proteins are identified by UniProt protein (gene) symbol.

Figure 5. Keratins upregulated in response to Aquamin ${ }^{\circledR}$. Values represent average fold-change from high-fat control based on $n=5$ liver samples per treatment group. Corresponding values for response to low-fat diet and response to OCA are shown for comparison. 
Table 1A. Significantly upregulated proteins with Aquamin in high-fat mice

\begin{tabular}{lllll} 
& & \multicolumn{2}{c}{ MS-NASH } & C57BL6 \\
Proteins & Genes & Aquamin & OCA & (Low-Fat) \\
\cline { 3 - 5 } UDP-N-acetylhexosamine pyrophosphorylase-like & & & & $1.1 \pm 0.2$ \\
protein 1 & Uap1l1 & $1.9 \pm 0.8^{*}$ & $0.9 \pm 0.4$ & $0.8 \pm 0.3$ \\
Biglycan & Bgn & $1.7 \pm 0.5^{*}$ & $1.5 \pm 0.6$ & $1.1 \pm 0.5$ \\
Tax1-binding protein 3 & Tax1bp3 & $1.6 \pm 0.5^{*}$ & $1.6 \pm 1.1$ & \\
Succinyl-CoA:3-ketoacid coenzyme A transferase 1, & & & & \\
mitochondrial & Oxct1 & $1.6 \pm 0.3^{*}$ & $1.4 \pm 0.2^{*}$ & $1.2 \pm 0.4$ \\
Glycogen phosphorylase, brain form & Pygb & $1.5 \pm 0.3^{*}$ & $0.9 \pm 0.2$ & $0.6 \pm 0.1$ \\
Acyl-coenzyme A thioesterase 9, mitochondrial & Acot9 & $1.5 \pm 0.4^{*}$ & $1.3 \pm 0.6$ & $0.8 \pm 0.6$ \\
CD166 antigen & Alcam & $1.5 \pm 0.4^{*}$ & $1.3 \pm 0.5$ & $1.2 \pm 0.2^{*}$ \\
Erythrocyte band 7 integral membrane protein & Stom & $1.5 \pm 0.4^{*}$ & $1.2 \pm 0.5$ & $0.9 \pm 0.5$ \\
RNA-binding protein 3 & Rbm3 & $1.5 \pm 0.3^{*}$ & $1.4 \pm 0.4^{*}$ & $1.2 \pm 0.3$ \\
Angiotensinogen & Agt & $1.5 \pm 0.4^{*}$ & $1.1 \pm 0.3$ & $1.5 \pm 0.4^{*}$ \\
Apolipoprotein A-I & Apoa1 & $1.5 \pm 0.3^{*}$ & $1.0 \pm 0.3$ & $0.8 \pm 0.2$ \\
Heparin cofactor 2 & Serpind1 & $1.5 \pm 0.4^{*}$ & $1.2 \pm 0.6$ & $1.1 \pm 0.4$ \\
\hline
\end{tabular}

These values (in Table $1 \mathrm{~A}$ ) represent average ( \pm standard deviation) fold-change of abundance ratios for each altered (upregulated) protein compared to the high-fat control group (MS-NASH mice on a high-fat diet) with a 1.5-fold change threshold in response to Aquamin intervention and are significant with a p-value $<0.05\left(^{*}\right)$. For each upregulated protein with Aquamin, corresponding values from the other two groups are shown for comparison. These liver samples (from 5 mice in each group) were individually assessed by TMT-based differential proteomic expression and data were merged to get averages. Protein FDR Confidence for all proteins was $<1 \%$. Asterisks indicate statistical significance as compared to High-fat control at $p<0.05$. FDR: False Discovery Rate. These data are also presented in Figure 4.

Table 1B. Top pathways associated with upregulated proteins (listed in 1A) altered with Aquamin

Pathway name

HDL clearance

Scavenging by Class B Receptors

Utilization of Ketone Bodies

RHO GTPases Activate Rhotekin and Rhophilins

HDL assembly

Post-translational protein phosphorylation

Regulation of IGF transport and uptake by IGFBPs

HDL remodeling

Chylomicron remodeling

Chylomicron assembly

Scavenging by Class A Receptors

Ketone body metabolism

Dermatan sulfate biosynthesis

Heme signaling

CS/DS degradation

Metabolism of Angiotensinogen to Angiotensins

$A B C$ transporters in lipid homeostasis

Plasma lipoprotein assembly

Chondroitin sulfate biosynthesis

Common Pathway of Fibrin Clot Formation

Intrinsic Pathway of Fibrin Clot Formation

A tetrasaccharide linker sequence is required for GAG synthesis
Entities

pValue

0.004

0.005

0.005

0.008

0.009

0.010

0.011

0.012

0.013

0.013

0.013

0.013

0.014

0.014

0.018

0.021

0.022

0.024

0.026

0.027

0.030

0.034
Mapped entities

Apoa1

Apoa1

Oxct1

Tax1bp3

Apoa1

Apoa1;Serpind1

Apoa1;Serpind1

Apoa1

Apoa1

Apoa1

Apoa1

Oxct1

Bgn

Apoa1

Bgn

Agt

Apoa1

Apoa1

Bgn

Serpind1

Serpind1

Bgn 


$\begin{array}{lll}\text { Plasma lipoprotein remodeling } & 0.035 & \text { Apoa1 } \\ \text { Plasma lipoprotein clearance } & 0.039 & \text { Apoa1 } \\ \text { RHOH GTPase cycle } & 0.048 & \text { Stom } \\ \text { Mitochondrial Fatty Acid Beta-Oxidation } & 0.048 & \text { Acot9 } \\ \text { Formation of Fibrin Clot (Clotting Cascade) } & 0.049 & \text { Serpind1 }\end{array}$

The pathways listed here are altered by the significantly upregulated proteins with the intervention "Aquamin" presented in Table 1A. Reactome (v78) was used to generate the pathway analysis report for species Mus musculus. The significance ( $p$-value) is calculated by the overrepresentation analysis (hypergeometric distribution). IGF: Insulin-like Growth Factor, IGFBPs: Insulin-like growth factor binding proteins

Table 1C. Significantly downregulated proteins with Aquamin in high-fat mice

\begin{tabular}{|c|c|c|c|c|}
\hline \multirow[b]{2}{*}{ Proteins } & \multirow[b]{2}{*}{ Genes } & \multicolumn{2}{|c|}{ MS-NASH } & \multirow{2}{*}{$\begin{array}{l}\text { C57BL6 } \\
\text { (Low-Fat) }\end{array}$} \\
\hline & & Aquamin & OCA & \\
\hline Cytochrome P450 2C54 & Cyp2c54 & $0.48 \pm 0.14^{*}$ & $0.61 \pm 0.19^{*}$ & $2.72 \pm 0.49$ \\
\hline $\begin{array}{l}\text { Major intrinsically disordered NOTCH2-binding } \\
\text { receptor 1-like homolog }{ }^{\#}\end{array}$ & Minar2 & $0.49 \pm 0.12^{*}$ & $0.45 \pm 0.18^{*}$ & $1.06 \pm 0.23$ \\
\hline Alpha-1-antitrypsin 1-5 & Serpina1e & $0.52 \pm 0.27^{*}$ & $0.70 \pm 0.35$ & $37.05 \pm 11.87$ \\
\hline Protein CASC3 & Casc3 & $0.57 \pm 0.13^{*}$ & $0.55 \pm 0.16^{*}$ & $0.60 \pm 0.22^{*}$ \\
\hline Major urinary protein 2 & Mup2 & $0.59 \pm 0.23^{*}$ & $0.44 \pm 0.19^{*}$ & $10.40 \pm 5.30$ \\
\hline Cysteine dioxygenase type 1 & Cdo1 & $0.59 \pm 0.18^{*}$ & $0.54 \pm 0.11^{*}$ & $1.94 \pm 0.66$ \\
\hline Sodium-coupled neutral amino acid transporter 4 & Slc38a4 & $0.60 \pm 0.27^{*}$ & $0.62 \pm 0.27^{*}$ & $1.30 \pm 0.37$ \\
\hline Ferritin light chain 1 & $\mathrm{Ftl1}$ & $0.62 \pm 0.23^{*}$ & $0.74 \pm 0.27$ & $1.18 \pm 0.80$ \\
\hline $40 S$ ribosomal protein $\mathrm{S} 30$ & Fau & $0.63 \pm 0.33^{*}$ & $0.71 \pm 0.26^{*}$ & $0.80 \pm 0.42$ \\
\hline $\begin{array}{l}\text { Sodium-coupled neutral amino acid transporter } 3 \\
\text { 2-oxo-4-hydroxy-4-carboxy-5-ureidoimidazoline }\end{array}$ & Slc38a3 & $0.63 \pm 0.13^{*}$ & $0.71 \pm 0.18^{*}$ & $2.60 \pm 1.10$ \\
\hline decarboxylase & Urad & $0.64 \pm 0.20^{*}$ & $0.94 \pm 0.20$ & $3.19 \pm 2.07$ \\
\hline Cytochrome P450 2C50 & Сyp2c50 & $0.65 \pm 0.22^{*}$ & $0.79 \pm 0.40$ & $4.82 \pm 2.53$ \\
\hline 5'-nucleotidase & Nt5e & $0.66 \pm 0.28^{*}$ & $0.67 \pm 0.19^{*}$ & $1.33 \pm 0.30$ \\
\hline Ileal sodium/bile acid cotransporter & Slc10a2 & $0.67 \pm 0.23^{*}$ & $0.77 \pm 0.22$ & $0.54 \pm 0.27^{*}$ \\
\hline
\end{tabular}

These values (in Table 1C) represent average ( \pm standard deviation) fold-change of abundance ratios for each altered (downregulated) protein compared to the high-fat control group (MS-NASH mice on a high-fat) with a 1.5-fold change threshold in response to Aquamin intervention and are significant with a p-value $<0.05\left(^{*}\right)$. For each downregulated protein with Aquamin, corresponding values from the other two groups are shown for comparison. These liver samples (from 5 mice in each group) were individually assessed by TMT-based differential proteomic expression and data were merged to get averages. Protein FDR Confidence for all proteins was $<1 \%$ except for 1 downregulated protein\# $(\leq 2 \%)$. Asterisks indicate statistical significance as compared to High-fat control at $p<0.05$. FDR: False Discovery Rate. These data are also presented in Figure 4.

Table 1D. Top pathways associated with downregulated proteins (listed in 1C) altered with Aquamin Entities

Amino acid transport across the plasma membrane pValue Mapped entities

Transport of inorganic cations/anions and amino acids/oligopeptides 0.001 Pyrimidine catabolism 0.011

Degradation of cysteine and homocysteine

$0.017 \quad \mathrm{Nt5e}$

Recycling of bile acids and salts

0.018 Slc38a3;SIc38a4

Purine catabolism $0.026 \quad$ Slc10a2

Sulfur amino acid metabolism $0.027 \quad \mathrm{Nt} 5 \mathrm{e}$ Nicotinate metabolism $0.035 \quad$ Cdo1 SLC-mediated transmembrane transport $0.047 \quad \mathrm{Nt5e}$ Nucleotide catabolism $0.050 \quad$ Slc38a3;Slc38a4 $0.050 \quad \mathrm{Nt5e}$ 
bioRxiv preprint doi: https://doi.org/10.1101/2022.01.29.478332; this version posted January 30, 2022. The copyright holder for this preprint (which was not certified by peer review) is the author/funder. All rights reserved. No reuse allowed without permission.

The pathways listed here are altered by the significantly downregulated proteins with the intervention "Aquamin" presented in Table 1C. Reactome (v78) was used to generate the pathway analysis report for species Mus musculus. The significance ( $p$-value) is calculated by the overrepresentation analysis (hypergeometric distribution). 
Table 2. Upregulated proteins by an unbiased proteomic screening with Aquamin in high-fat mice

\begin{tabular}{|c|c|c|c|c|}
\hline \multirow[b]{2}{*}{ Proteins } & \multirow[b]{2}{*}{ Genes } & \multicolumn{2}{|c|}{ MS-NASH } & \multirow{2}{*}{$\begin{array}{l}\text { C57BL6 } \\
\text { Low-Fat }\end{array}$} \\
\hline & & Aquamin & OCA & \\
\hline Keratin, type II cytoskeletal 79 & Krt79 & $26.34 \pm 43.11$ & $5.73 \pm 7.97$ & $5.40 \pm 4.07$ \\
\hline Nuclear transport factor 2 & Nutf2 & $12.12 \pm 24.59$ & $10.29 \pm 20.76$ & $8.16 \pm 16.12$ \\
\hline Keratin, type II cytoskeletal 5 & Krt5 & $11.14 \pm 22.14$ & $1.60 \pm 2.45$ & $3.56 \pm 4.11$ \\
\hline Keratin, type I cytoskeletal 17 & Krt17 & $10.62 \pm 21.47$ & $1.23 \pm 1.59$ & $3.50 \pm 2.69$ \\
\hline Keratin, type II cytoskeletal 1 & Krt1 & $10.38 \pm 20.44$ & $1.80 \pm 2.51$ & $3.96 \pm 3.36$ \\
\hline Fucose mutarotase & Fuom & $10.28 \pm 20.61$ & $10.03 \pm 19.94$ & $8.72 \pm 17.40$ \\
\hline $\mathrm{SH} 3$ domain-containing protein $21^{\#}$ & Sh3d21 & $8.30 \pm 16.34$ & $5.43 \pm 10.12$ & $6.05 \pm 10.62$ \\
\hline Keratin, type II cytoskeletal 2 oral & Krt76 & $8.07 \pm 14.08$ & $1.71 \pm 2.42$ & $5.89 \pm 7.01$ \\
\hline Keratin, type I cytoskeletal 42 & Krt42 & $7.91 \pm 13.57$ & $1.26 \pm 1.63$ & $3.74 \pm 3.98$ \\
\hline Glycine cleavage system $\mathrm{H}$ protein, mitochondrial & Gcsh & $7.63 \pm 14.93$ & $9.05 \pm 17.88$ & $11.09 \pm 21.41$ \\
\hline Pigment epithelium-derived factor & Serpinf1 & $6.03 \pm 10.85$ & $5.58 \pm 10.41$ & $3.34 \pm 5.88$ \\
\hline Tubulin beta- 5 chain & Tubb5 & $5.81 \pm 10.51$ & $5.06 \pm 9.36$ & $4.90 \pm 9.25$ \\
\hline Keratin, type I cytoskeletal 14 & Krt14 & $5.58 \pm 9.45$ & $0.70 \pm 0.43$ & $5.88 \pm 7.13$ \\
\hline Transmembrane protein 14C & Tmem14c & $5.56 \pm 10.29$ & $4.82 \pm 8.80$ & $5.97 \pm 10.43$ \\
\hline Ras-related protein R-Ras & Rras & $5.46 \pm 9.57$ & $4.75 \pm 8.44$ & $6.32 \pm 11.74$ \\
\hline Ras-related protein Rap-1A $A^{\#}$ & Rap1a & $5.20 \pm 9.18$ & $5.83 \pm 10.73$ & $5.66 \pm 10.21$ \\
\hline Protein PAT1 homolog 1 & Patl1 & $4.99 \pm 4.23$ & $2.74 \pm 2.40$ & $3.09 \pm 2.72$ \\
\hline Keratin, type I cytoskeletal 10 & Krt10 & $4.86 \pm 8.83$ & $1.99 \pm 3.15$ & $4.10 \pm 6.43$ \\
\hline Tubulin beta-4A chain & Tubb4a & $4.85 \pm 8.51$ & $4.73 \pm 8.30$ & $3.76 \pm 6.90$ \\
\hline Keratin, type II cytoskeletal 2 epidermal & Krt2 & $4.58 \pm 8.23$ & $1.47 \pm 1.86$ & $2.15 \pm 1.84$ \\
\hline $60 S$ ribosomal protein L36 & Rpl36 & $4.32 \pm 7.31$ & $4.95 \pm 8.52$ & $4.35 \pm 8.31$ \\
\hline Keratin, type I cytoskeletal 16 & Krt16 & $4.30 \pm 6.06$ & $0.80 \pm 0.58$ & $2.02 \pm 1.83$ \\
\hline IgG receptor FcRn large subunit p51 & Fcgrt & $4.12 \pm 6.83$ & $5.74 \pm 10.39$ & $4.17 \pm 6.07$ \\
\hline U8 snoRNA-decapping enzyme & Nudt16 & $4.03 \pm 5.49$ & $3.02 \pm 4.24$ & $3.26 \pm 4.15$ \\
\hline Ig kappa chain V-V region MOPC 149 & $\operatorname{lgkv12-44}$ & $4.02 \pm 4.71$ & $2.06 \pm 2.70$ & $1.52 \pm 2.10$ \\
\hline Alpha-1-acid glycoprotein 1 & Orm1 & $3.98 \pm 6.98$ & $2.93 \pm 5.05$ & $6.12 \pm 9.25$ \\
\hline Endophilin-B1 & Sh3glb1 & $3.93 \pm 6.42$ & $3.47 \pm 5.80$ & $3.27 \pm 5.18$ \\
\hline Proteasome subunit beta type- 3 & Psmb3 & $3.56 \pm 5.56$ & $3.50 \pm 5.41$ & $3.27 \pm 4.30$ \\
\hline $60 S$ acidic ribosomal protein $\mathrm{P} 1$ & Rplp1 & $3.53 \pm 5.59$ & $3.61 \pm 5.97$ & $4.84 \pm 8.27$ \\
\hline Coatomer subunit epsilon & Cope & $3.50 \pm 5.59$ & $2.78 \pm 4.10$ & $3.27 \pm 4.74$ \\
\hline Copper transport protein ATOX1 & Atox1 & $3.44 \pm 5.40$ & $2.84 \pm 4.30$ & $1.64 \pm 2.28$ \\
\hline Translation initiation factor elF-2B subunit epsilon & Eif2b5 & $3.42 \pm 4.73$ & $1.63 \pm 1.08$ & $1.76 \pm 1.80$ \\
\hline Galectin-related protein & Lgalsl & $3.26 \pm 5.14$ & $3.52 \pm 5.77$ & $2.86 \pm 4.63$ \\
\hline Putative RNA-binding protein Luc7-like 1 & Luc7l & $3.20 \pm 4.78$ & $2.56 \pm 3.40$ & $2.67 \pm 3.04$ \\
\hline 5'-AMP-activated protein kinase subunit beta-1 & Prkab1 & $3.05 \pm 4.30$ & $3.00 \pm 3.74$ & $1.92 \pm 2.61$ \\
\hline Splicing factor 3B subunit 4 & Sf3b4 & $3.03 \pm 4.20$ & $3.57 \pm 5.19$ & $3.46 \pm 4.27$ \\
\hline 2-hydroxyacyl-CoA lyase 2 & llvbl & $2.99 \pm 4.38$ & $2.82 \pm 4.22$ & $2.91 \pm 4.43$ \\
\hline Small nuclear ribonucleoprotein $\mathrm{E}$ & Snrpe & $2.90 \pm 4.12$ & $2.22 \pm 2.99$ & $2.86 \pm 3.61$ \\
\hline Arylsulfatase A & Arsa & $2.76 \pm 3.37$ & $1.06 \pm 0.95$ & $0.81 \pm 0.40$ \\
\hline Aldose reductase-related protein 2 & Akr1b8 & $2.75 \pm 3.39$ & $2.25 \pm 2.73$ & $2.84 \pm 3.75$ \\
\hline Aldo-keto reductase family 1 member $\mathrm{C} 18$ & Akr1c18 & $2.74 \pm 3.86$ & $2.31 \pm 2.65$ & $2.52 \pm 2.51$ \\
\hline H-2 class II histocompatibility antigen gamma chain & $\mathrm{Cd} 74$ & $2.73 \pm 3.74$ & $0.97 \pm 0.77$ & $0.47 \pm 0.18$ \\
\hline $\begin{array}{l}\text { Ig gamma-2A chain } \mathrm{C} \text { region secreted form } \\
\text { S-methylmethionine--homocysteine S-methyltransferase }\end{array}$ & lghg2a & $2.70 \pm 2.55$ & $1.17 \pm 0.49$ & $0.72 \pm 0.32$ \\
\hline BHMT2 & Bhmt2 & $2.70 \pm 3.60$ & $2.51 \pm 3.26$ & $2.01 \pm 2.67$ \\
\hline
\end{tabular}


Glutaredoxin-1 protein 1

Nuclear cap-binding protein subunit 1

NAD-dependent malic enzyme, mitochondrial

Cytochrome b-245 heavy chain

Peptidyl-prolyl cis-trans isomerase NIMA-interacting 1

Signal transducer and activator of transcription 1

Protein quaking

Cytochrome b-c1 complex subunit 10

Signal recognition particle $19 \mathrm{kDa}$ protein

Fermitin family homolog 3

AP-2 complex subunit sigma

Platelet-activating factor acetylhydrolase IB subunit

gamma

Neuroplastin

$\mathrm{NADH}$-ubiquinone oxidoreductase chain 5

Guanylate-binding protein 1

Carboxypeptidase B2

Lysosomal acid lipase/cholesteryl ester hydrolase

[Protein ADP-ribosylarginine] hydrolase

cAMP-dependent protein kinase type I-alpha regulatory

subunit

ADP-ribosylation factor 4

Tubulin beta-2A chain

COMM domain-containing protein 8

$\mathrm{H}-2$ class II histocompatibility antigen, I-A beta chain

Rho guanine nucleotide exchange factor 10 -like protein

TIP41-like protein

Galactose-1-phosphate uridylyltransferase

Acetyl-coenzyme A transporter 1

Nascent polypeptide-associated complex subunit alpha,

muscle-specific

Vascular non-inflammatory molecule 3

Protein S100-A11

CD81 antigen

$\mathrm{H}-2$ class II histocompatibility antigen, A-B alpha chain

Charged multivesicular body protein $1 \mathrm{~b}-1$

STIP1 homology and $U$ box-containing protein 1

Proteasome subunit beta type-8

Acyl-CoA-binding protein

Serine hydrolase-like protein ${ }^{\#}$

WD repeat-containing protein 18

Small nuclear ribonucleoprotein Sm D1

Lysosomal acid phosphatase

Ancient ubiquitous protein 1
LIM and senescent cell antigen-like-containing domain

\begin{tabular}{|c|c|c|c|}
\hline GIrx & $2.58 \pm 3.23$ & $2.85 \pm 3.94$ & $3.76 \pm 5.34$ \\
\hline Lims1 & $2.55 \pm 3.39$ & $1.55 \pm 1.45$ & $1.37 \pm 1.22$ \\
\hline Ncbp1 & $2.54 \pm 3.24$ & $2.41 \pm 3.23$ & $2.29 \pm 2.99$ \\
\hline Me2 & $2.54 \pm 3.24$ & $1.95 \pm 2.35$ & $2.24 \pm 2.73$ \\
\hline Cybb & $2.53 \pm 3.03$ & $1.82 \pm 2.05$ & $1.42 \pm 1.62$ \\
\hline Pin1 & $2.52 \pm 2.87$ & $2.51 \pm 2.90$ & $2.43 \pm 2.54$ \\
\hline Stat1 & $2.51 \pm 2.96$ & $1.51 \pm 1.49$ & $0.66 \pm 0.30$ \\
\hline Qki & $2.49 \pm 3.17$ & $2.06 \pm 2.46$ & $1.99 \pm 2.52$ \\
\hline Uqcr11 & $2.47 \pm 3.24$ & $2.45 \pm 3.24$ & $3.40 \pm 4.36$ \\
\hline Srp19 & $2.46 \pm 3.39$ & $2.29 \pm 3.07$ & $3.08 \pm 4.09$ \\
\hline Fermt3 & $2.46 \pm 2.45$ & $1.51 \pm 1.48$ & $1.30 \pm 1.15$ \\
\hline Ap2s1 & $2.45 \pm 3.06$ & $2.05 \pm 2.57$ & $2.61 \pm 3.59$ \\
\hline Pafah1b3 & $2.45 \pm 1.29$ & $2.21 \pm 1.34$ & $1.80 \pm 0.90$ \\
\hline Nptn & $2.44 \pm 3.07$ & $2.11 \pm 2.67$ & $2.33 \pm 2.72$ \\
\hline Mtnd5 & $2.42 \pm 3.25$ & $2.44 \pm 3.18$ & $2.55 \pm 3.40$ \\
\hline Gbp1 & $2.40 \pm 3.98$ & $1.69 \pm 2.15$ & $0.58 \pm 0.28$ \\
\hline Cpb2 & $2.39 \pm 2.63$ & $2.29 \pm 2.74$ & $3.39 \pm 4.66$ \\
\hline Lipa & $2.38 \pm 2.64$ & $1.68 \pm 1.83$ & $1.89 \pm 2.16$ \\
\hline Adprh & $2.36 \pm 2.84$ & $2.05 \pm 2.29$ & $1.53 \pm 1.69$ \\
\hline Prkar1a & $2.31 \pm 2.86$ & $1.96 \pm 2.45$ & $1.76 \pm 1.95$ \\
\hline Arf4 & $2.31 \pm 2.87$ & $2.02 \pm 2.64$ & $1.84 \pm 2.57$ \\
\hline Tubb2a & $2.30 \pm 2.80$ & $2.26 \pm 2.77$ & $1.00 \pm 1.29$ \\
\hline Commd8 & $2.26 \pm 2.73$ & $2.16 \pm 1.99$ & $2.63 \pm 2.14$ \\
\hline H2-Eb1 & $2.22 \pm 2.52$ & $1.26 \pm 0.70$ & $0.54 \pm 0.33$ \\
\hline Arhgef10l & $2.19 \pm 2.68$ & $1.90 \pm 2.04$ & $1.43 \pm 1.52$ \\
\hline Tiprl & $2.18 \pm 2.36$ & $2.36 \pm 2.70$ & $1.63 \pm 1.88$ \\
\hline Galt & $2.14 \pm 2.67$ & $3.02 \pm 4.19$ & $2.84 \pm 3.21$ \\
\hline Slc33a1 & $2.13 \pm 2.62$ & $2.20 \pm 2.51$ & $2.04 \pm 2.75$ \\
\hline Naca & $2.13 \pm 2.58$ & $2.15 \pm 2.63$ & $2.29 \pm 2.60$ \\
\hline Vnn3 & $2.11 \pm 2.42$ & $1.75 \pm 2.17$ & $1.18 \pm 1.38$ \\
\hline S100a11 & $2.10 \pm 1.53$ & $1.17 \pm 1.17$ & $0.51 \pm 0.31$ \\
\hline Cd81 & $2.10 \pm 2.42$ & $1.67 \pm 2.05$ & $2.36 \pm 2.50$ \\
\hline $\mathrm{H} 2-\mathrm{Aa}$ & $2.09 \pm 1.00$ & $1.33 \pm 0.48$ & $0.62 \pm 0.13$ \\
\hline Chmp1b1 & $2.08 \pm 2.17$ & $1.81 \pm 1.87$ & $1.56 \pm 1.35$ \\
\hline Stub1 & $2.07 \pm 2.40$ & $2.22 \pm 2.68$ & $2.33 \pm 2.80$ \\
\hline Psmb8 & $2.06 \pm 1.71$ & $1.61 \pm 1.06$ & $1.10 \pm 0.63$ \\
\hline Dbi & $2.05 \pm 2.43$ & $2.20 \pm 3.02$ & $2.05 \pm 2.30$ \\
\hline Serhl & $2.05 \pm 1.88$ & $1.94 \pm 1.62$ & $2.10 \pm 2.09$ \\
\hline Wdr18 & $2.03 \pm 2.17$ & $2.18 \pm 2.66$ & $2.22 \pm 2.64$ \\
\hline Snrpd1 & $2.03 \pm 2.00$ & $2.05 \pm 2.04$ & $2.13 \pm 1.84$ \\
\hline Acp2 & $2.01 \pm 1.91$ & $1.45 \pm 1.20$ & $1.38 \pm 1.26$ \\
\hline Aup1 & $2.00 \pm 2.73$ & $2.04 \pm 2.78$ & $2.45 \pm 3.38$ \\
\hline
\end{tabular}

These values represent average ( \pm standard deviation) fold-change of abundance ratios for each altered protein compared to the high-fat control group (MS-NASH mice on a high-fat diet) with a 2-fold-change threshold in response to Aquamin intervention. For each upregulated protein with Aquamin, corresponding values from the other two groups are 
bioRxiv preprint doi: https://doi.org/10.1101/2022.01.29.478332; this version posted January 30, 2022. The copyright holder for this preprint (which was not certified by peer review) is the author/funder. All rights reserved. No reuse allowed without permission.

shown for comparison. The liver samples (from 5 mice in each group) were individually assessed by TMT-based differential proteomic expression and data were merged to get averages. Protein FDR Confidence for all proteins was $\leq 1 \%$ except for 3 upregulated proteins ${ }^{\#}(\leq 2 \%)$. FDR: False Discovery Rate. These data are also presented in Figure $3 A$. 
Table 3. Top pathways associated with upregulated proteins altered (at 2-fold change) with Aquamin

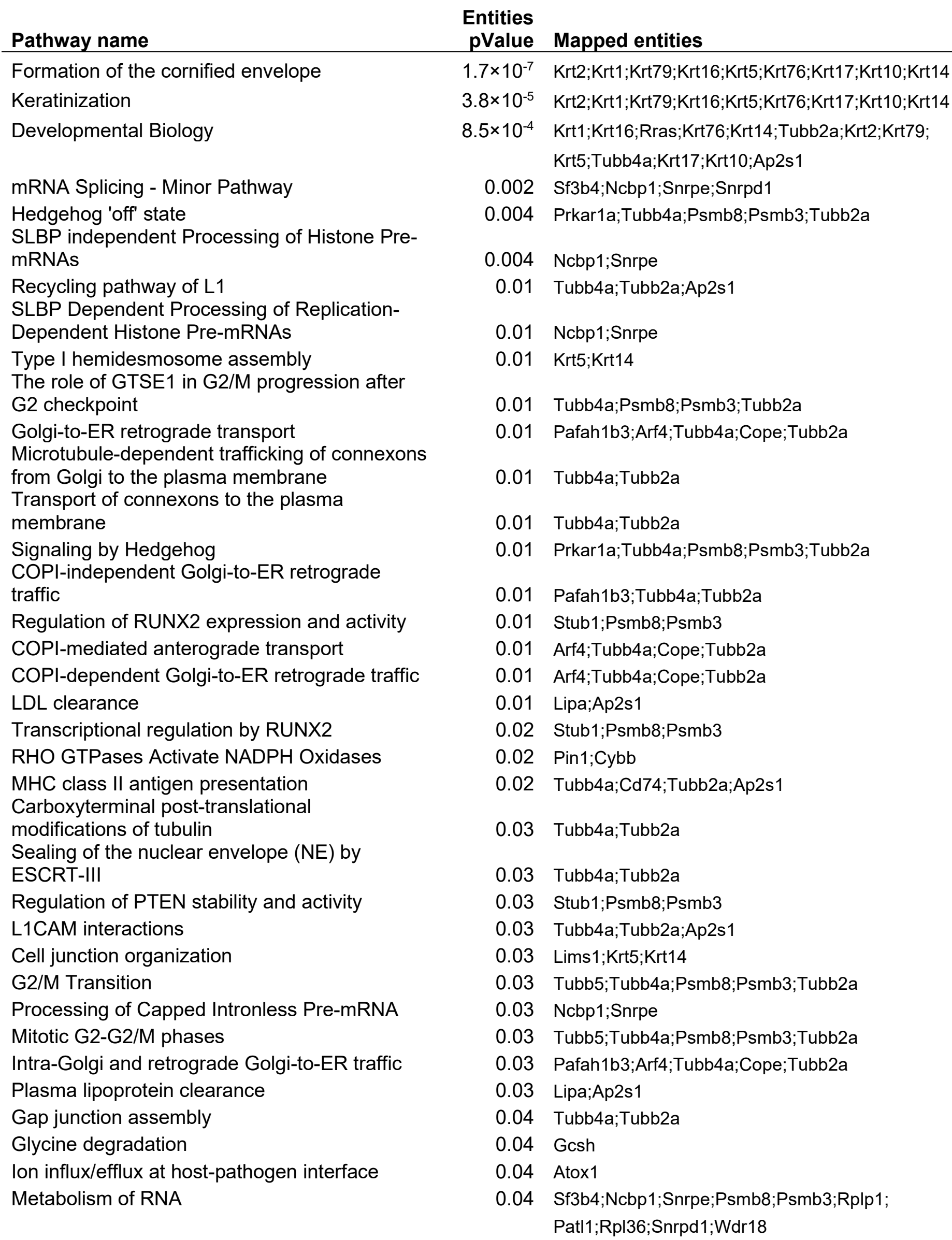


Aggrephagy

ARMS-mediated activation

Galactose catabolism

PCP/CE pathway

ER to Golgi Anterograde Transport

Recruitment of NuMA to mitotic centrosomes

SRP-dependent cotranslational protein

targeting to membrane

CREB1 phosphorylation through the activation

of Adenylate Cyclase

PKA activation in glucagon signalling

GDP-fucose biosynthesis

Cell-Cell communication

Gap junction trafficking

Gap junction trafficking and regulation
0.04 Tubb4a;Tubb2a
0.05 Rap1a
0.05 Galt
0.05 Psmb8;Psmb3;Ap2s1
0.05 Arf4;Tubb4a;Cope;Tubb2a
0.05 Tubb5;Tubb4a;Tubb2a
0.05 Srp19;Rplp1;Rpl36
0.06 Prkar1a
0.06 Prkar1a
0.06 Fuom
0.06 Lims1;Krt5;Krt14
0.06 Tubb4a;Tubb2a
0.06 Tubb4a;Tubb2a

The pathways listed here are altered by the upregulated proteins with the intervention "Aquamin" presented in Table 2. These proteins were selected by using an unbiased approach and 2-fold change threshold. Reactome (v78) was used to generate the pathway analysis report for species Mus musculus. The significance ( $p$-value) is calculated by the overrepresentation analysis (hypergeometric distribution). 
A.

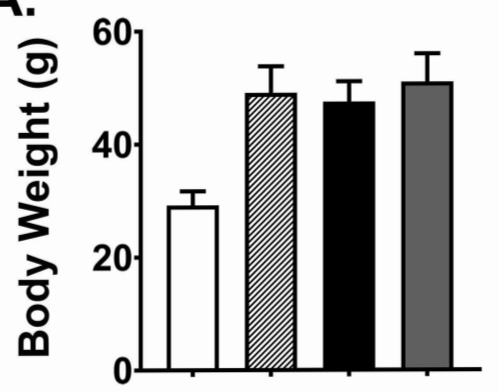

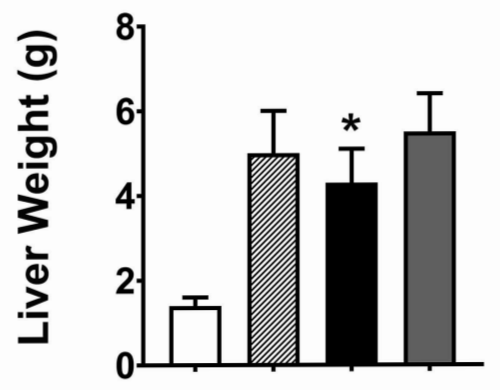

[ C57BL/6 (Low-Fat) 目 High-Fat (Control) I OCA (High-Fat )

口 Aquamin (High-Fat)
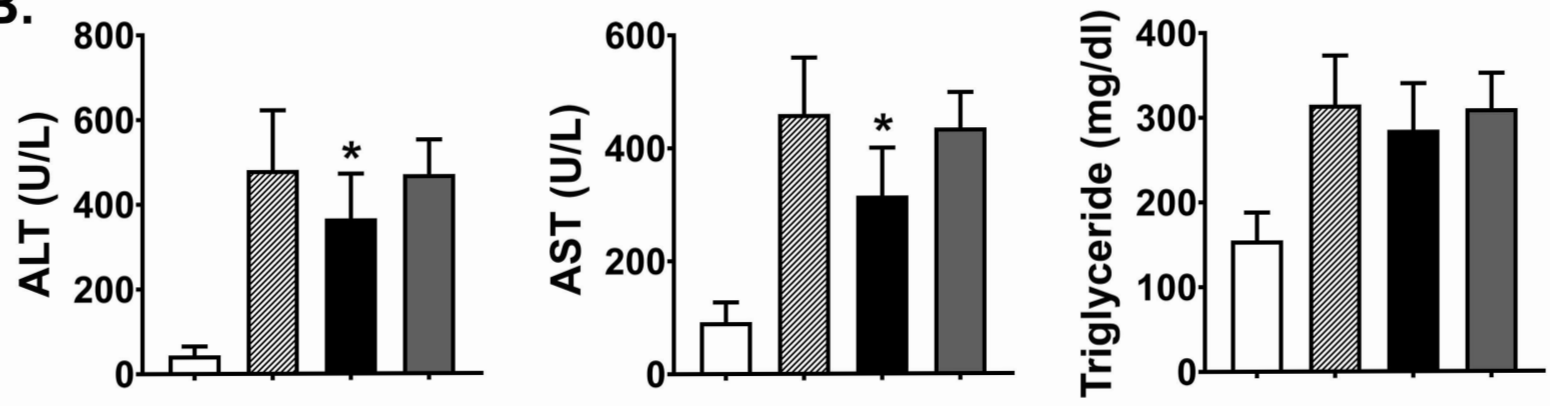
Figure 2
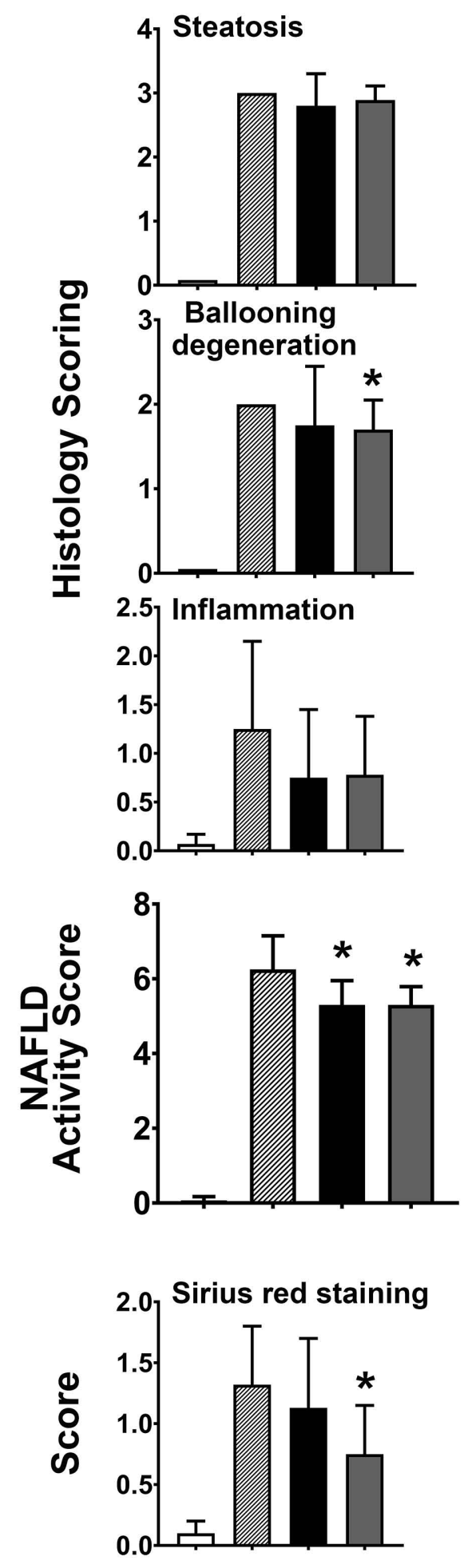

\section{C57BL6 (Low-Fat)}

MS-NASH (High-Fat)
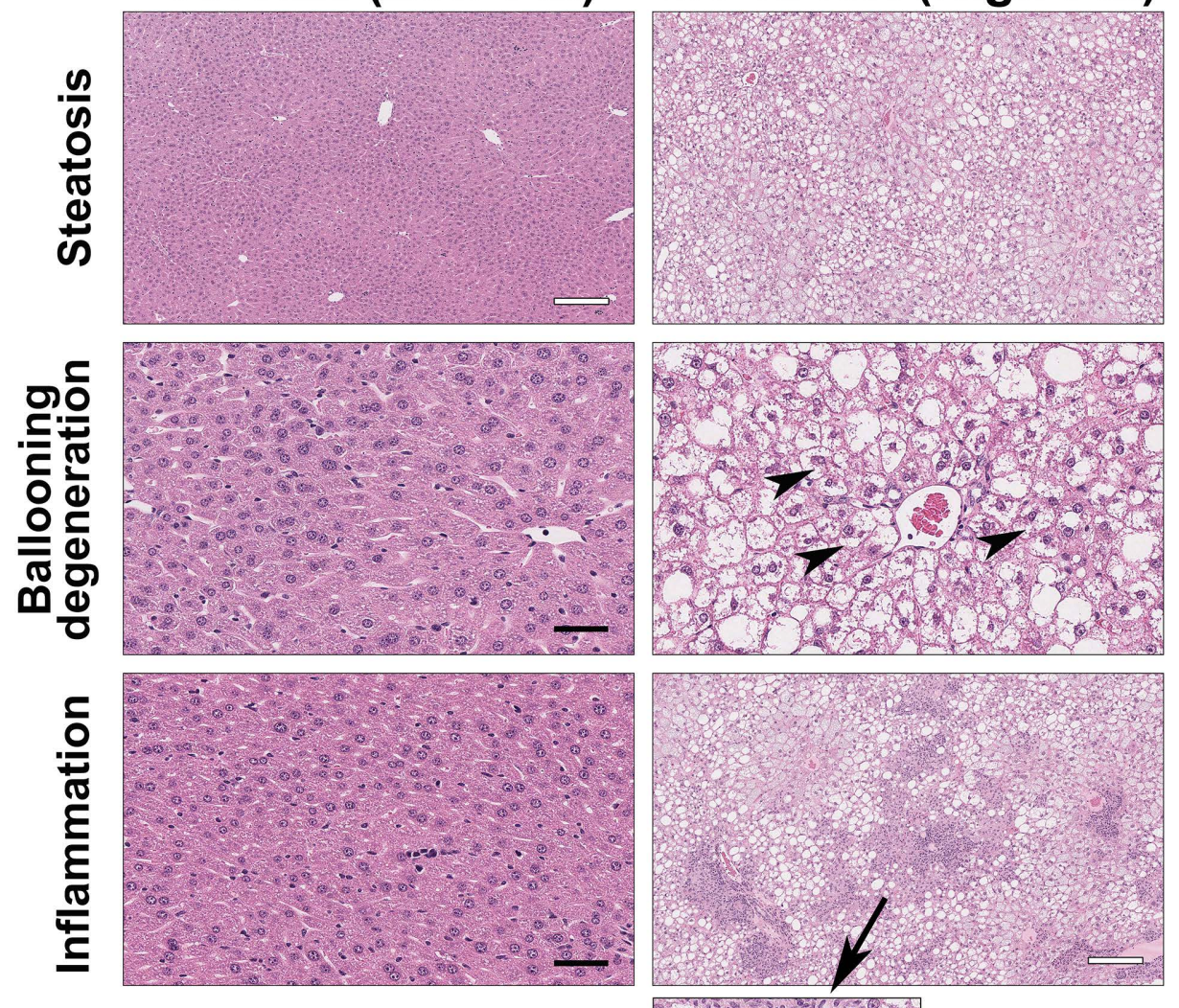

[ C57BL/6 (Low-Fat)

目High-Fat (Control)

OCA (High-Fat )

Aquamin (High-Fat)
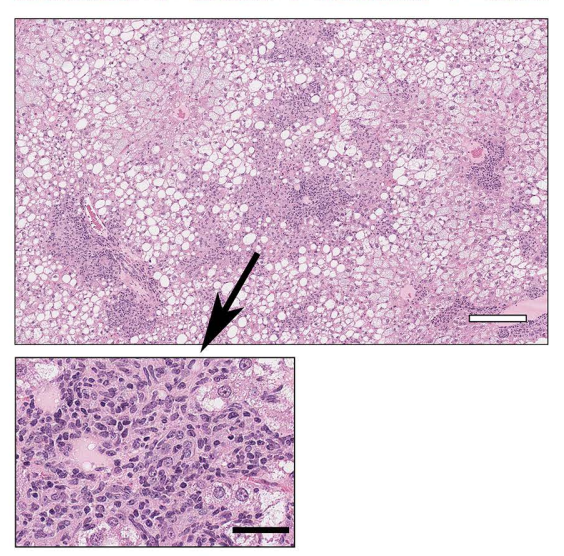
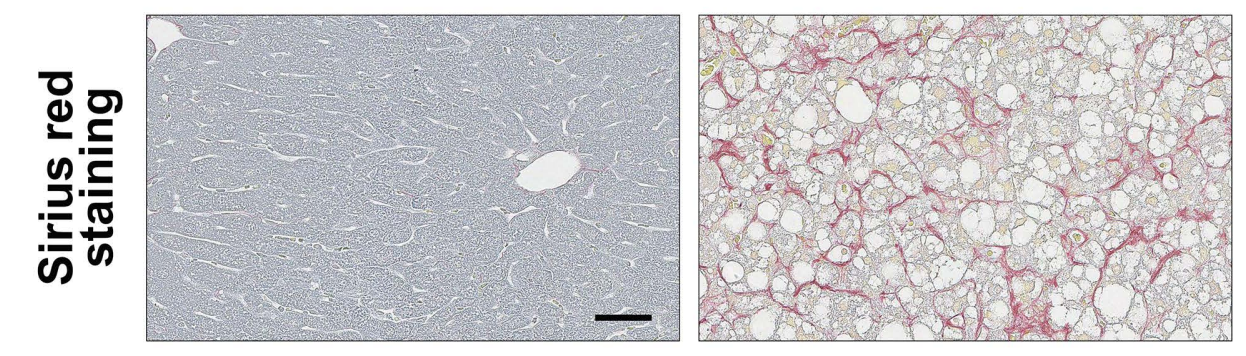
Figure 3

A. 2-Fold change

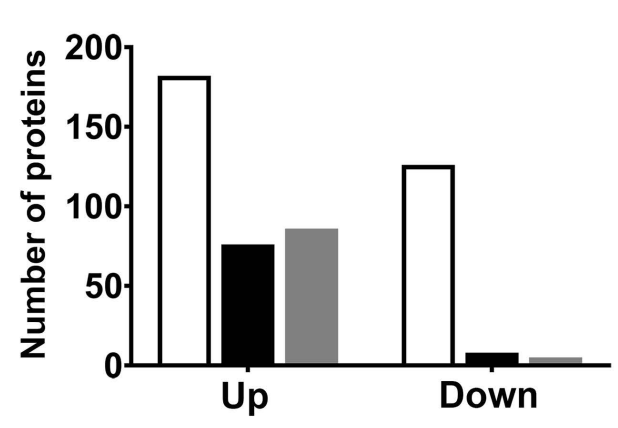

B. 1.5-Fold change ]C57BL/6 (Low-Fat)

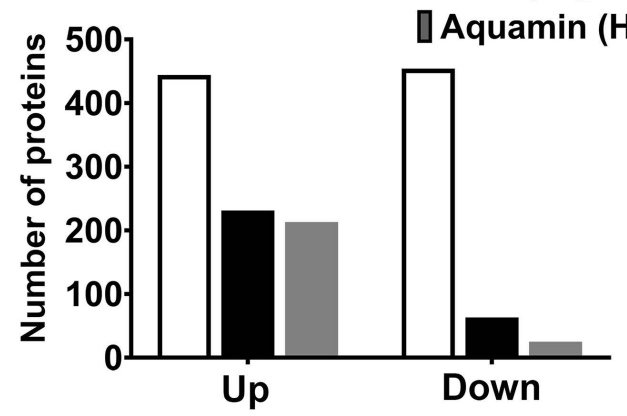

Upregulated

C57BL6 (LF)

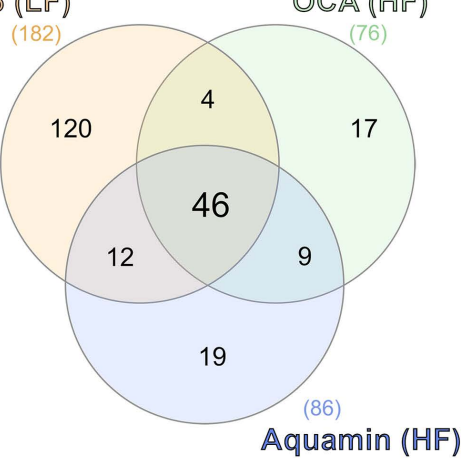

Upregulated

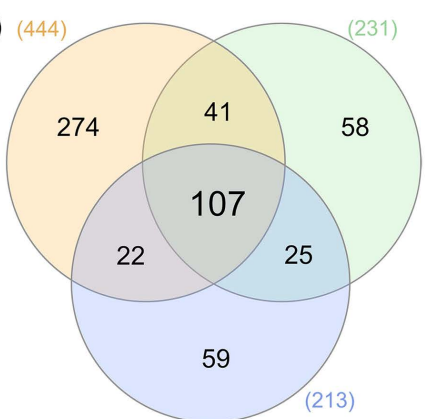

Downregulated

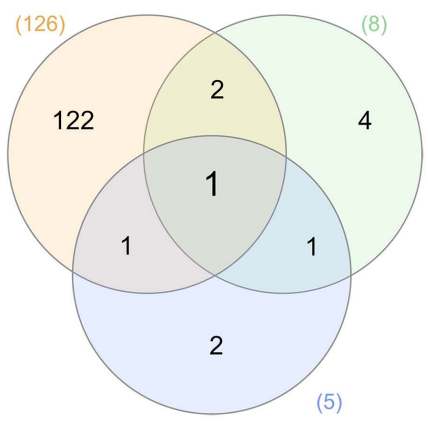

Downregulated

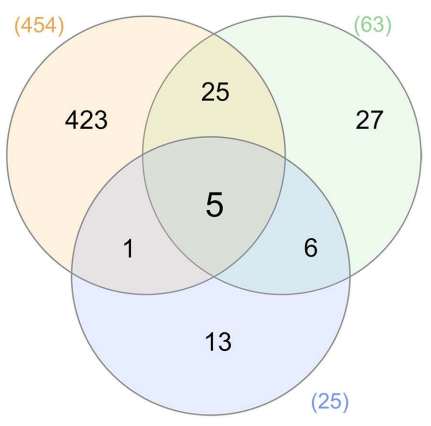




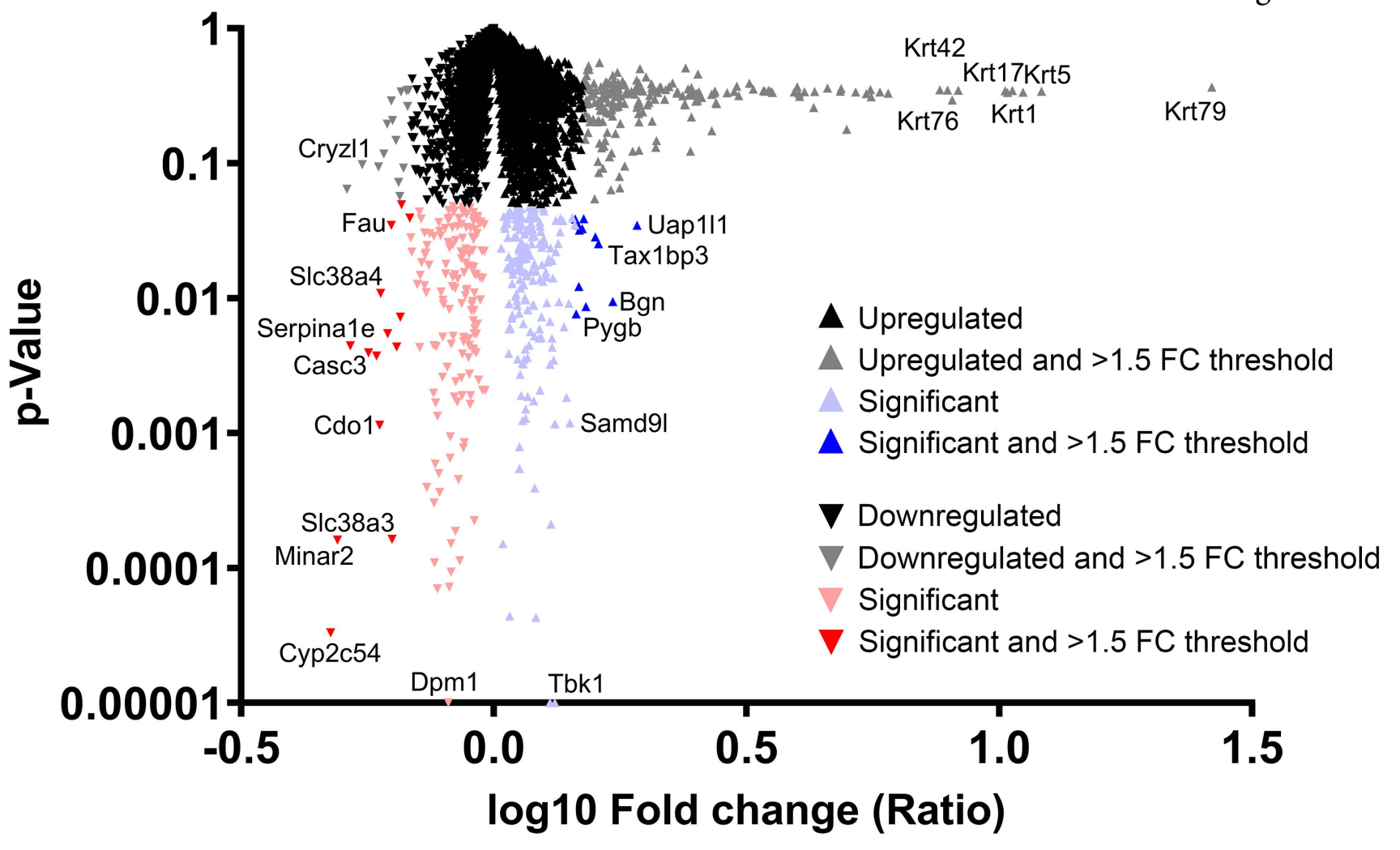


Figure 5

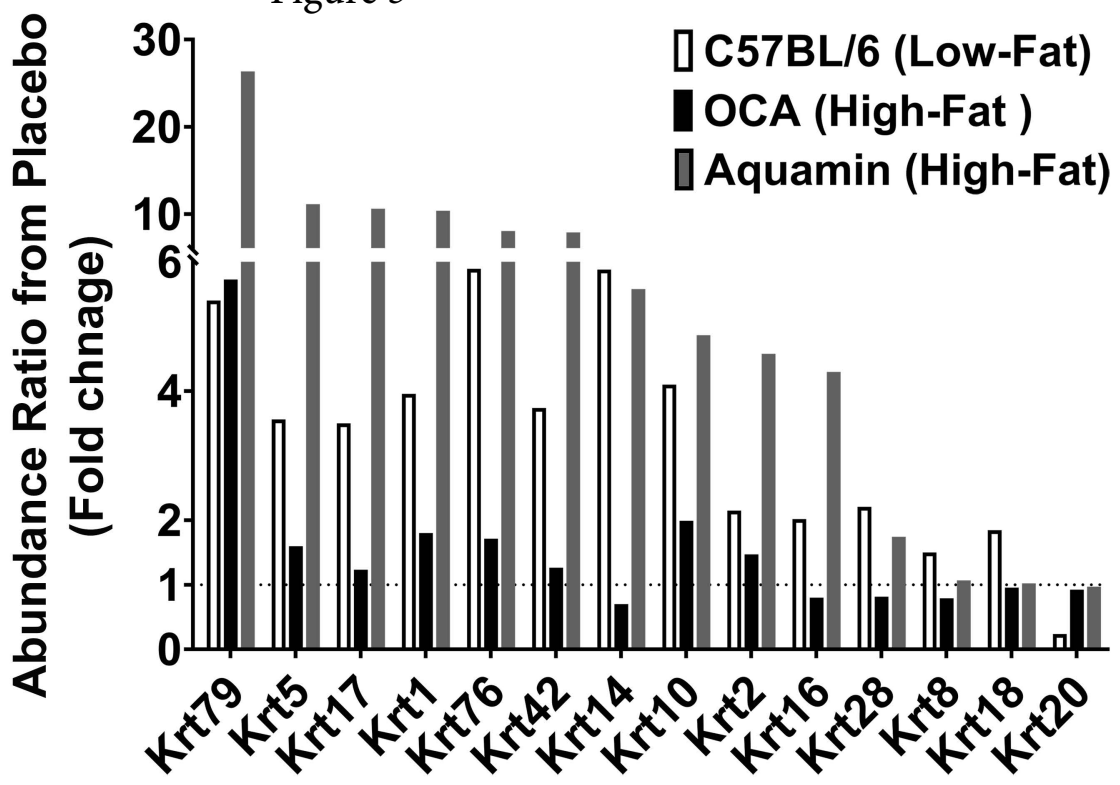

